\title{
VORTEX RINGS: EXISTENCE AND ASYMPTOTIC ESTIMATES
}

\author{
BY
}

AVNER FRIEDMAN ${ }^{1}$ AND BRUCE TURKINGTON

\begin{abstract}
ABSTRACr. The existence of a family of steady vortex rings is established by a variational principle. Further, the asymptotic behavior of the solutions is obtained for limiting values of an appropriate parameter $\lambda$; as $\lambda \rightarrow \infty$ the vortex ring tends to a torus whose cross-section is an infinitesimal disc.
\end{abstract}

0. Introduction. The study of steady vortex rings in an ideal fluid has been the subject of many investigations (see, for example, [3], [19] and the references given there). The classical examples are Helmholtz's rings of small cross-section [17] and Hill's spherical vortex [18].

A general existence theorem for vortex rings was first established by Fraenkel and Berger [13] (see also the very recent work [5], [20] with a similar approach); this paper also contains an excellent survey of the subject. The approach in [13] is based on a variational principle for the stream function.

More recently Benjamin [4] developed a new approach based on a variational principle for the vorticity. This approach is more natural since (i) the vorticity has compact support (whereas the stream function does not) and (ii) the quantities involved in the variational principle have direct physical significance.

In this paper we establish the existence of vortex rings by a new method. As in [4] we formulate the problem in a variational form for the kinetic energy as a functional of the vorticity. We take the admissible functions to vary in the set $Q_{\lambda}$ of functions $\zeta(x)$ satisfying:

$$
\begin{gathered}
\zeta(x)=\zeta(r, z)=\zeta(r,-z) \text { where } x=(r, \theta, z), \\
\frac{1}{2} \int r^{2} \zeta(x) d x=1, \quad \int \zeta(x) d x<1, \quad 0<\zeta(x)<\lambda,
\end{gathered}
$$

i.e., an axisymmetric flow with prescribed impulse $(=1)$, circulation $(<1)$ and vortex strength $(\leqslant \lambda)$; in [4] $\zeta$ is taken to vary over all rearrangements of a given function $\zeta_{0}(r, z)$. Our approach seems technically simpler; it has the further advantage that it leads to vortex rings with, essentially, any given vorticity function, such as

$$
\begin{array}{ll}
f(t)=c I_{\{t>0\}} & (c>0), \\
f(t)=c\left(t^{+}\right)^{\beta} & (c>0, \beta>0) .
\end{array}
$$

The method of solving our variational problem is in some sense an adaptation of the method of Auchmuty [1] and Auchmuty and Beals [2] (see also [14]-[16]) who

Received by the editors May 22, 1980.

AMS (MOS) subject classifications (1970). Primary 35J20, 76G05; Secondary 31A15, 35J05.

1 The first author is partially supported by National Science Foundation Grant MCS-781 7204. 
dealt with a variational problem for self-gravitating rotating fluids. There are, however, several differences, the most serious one being the nature of the constraints in (0.1).

Another object of this paper is to derive asymptotic estimates on the solution, which we shall denote by $\zeta=\zeta_{\lambda}$, as $\lambda \rightarrow \infty$. Denoting the support of $\zeta_{\lambda}(r, z)$ by $B_{\lambda}$, we prove

$$
\begin{aligned}
& E\left(\zeta_{\lambda}\right)=\left(1 / 8 \sqrt{2} \pi^{2}\right) \log \lambda+O(1)\left(E\left(\zeta_{\lambda}\right)=\text { kinetic energy }\right), \\
& c / \sqrt{\lambda} \leqslant \operatorname{diameter}\left(B_{\lambda}\right) \leqslant C / \sqrt{\lambda} \quad(0<c<C<\infty)
\end{aligned}
$$

as $\lambda \rightarrow \infty$, and

(0.6) $\quad B_{\lambda}$ is asymptotically a disc about $(\sqrt{2}, 0)$ with radius $1 /(\pi \sqrt{2 \lambda})$.

In $\S 1$ we give the physical background of the problem. In $\S 2$ we state the existence theorems in variational form for the vorticity, in cases $(0.2),(0.3)$. We also give an account of the relevant existence theorems in the literature. The existence of a vortex ring for the vorticity function $(0.3)$ is obtained in $\$ 4$. It is preceded by various estimates and some crucial energy identities which are derived in $\S 3$.

In $\$ 5$ we establish the existence of a vortex ring with the vorticity function $(0.2)$ by considering it as a limit case of $(0.3)$ with $\beta \rightarrow 0$; we were unable to treat the case $(0.2)$ more directly because of the nature of the constraints in (0.1).

In \$§6-8 asymptotic estimates are derived for $\lambda \rightarrow \infty$; we specialize here, for simplicity, to the case (0.2). In $\$ 6$ crude estimates are obtained on both $E\left(\zeta_{\lambda}\right)$ and on the support of $\zeta_{\lambda}$. The precise estimates $(0.4),(0.5)$ are established in $\$ 7$, using a capacity method recently developed by Caffarelli and Friedman [10]. Finally, (0.6) is proved in $\$ 8$.

Capacity methods have been recently introduced also by Berger and Fraenkel [6], [7]. Results of the form (0.4)-(0.6) have been proved by Fraenkel [11], [12] for vortex ring solutions defined by solving an integral equation with parameter $\lambda$. It is not known whether our solution, which is obtained by a variational principle, coincides with the solution of Fraenkel. In any case, the methods of Fraenkel and ours are entirely different.

1. Physical background. In this section we describe the equations governing the motion of a steady vortex ring in an ideal fluid and express the physical quantities involved in the form needed for the variational principle of the subsequent section.

Throughout the sequel we shall use the following notations: $x=(r, \theta, z)$ denotes the cylindrical coordinates of $x \in R^{3} ;\left\{\mathbf{i}_{r}, \mathbf{i}_{\theta}, \mathbf{i}_{z}\right\}$ represents the associated standard orthonormal frame; $d x=r d r d \theta d z$ denotes the volume element. Also, we shall write $d x$ for the measure $2 \pi r d r d z$ on the half-plane

$$
H=\{(r, z) ; 0 \leqslant r<\infty,-\infty<z<\infty\}
$$

The vortex ring is assumed to be steady, symmetric about the $z$-axis, and propagating with constant speed $W$ in the positive $z$-direction. With respect to axes fixed in the ring, the velocity field $\mathrm{v}(x), x \in R^{3}$, has the form

$$
\mathbf{v}(x)=v^{r}(r, z) \mathbf{i}_{r}+v^{z}(r, z) \mathbf{i}_{z},
$$




$$
\mathbf{v}(x) \rightarrow-W \mathbf{i}_{z} \quad \text { as }|x|=\left(r^{2}+z^{2}\right)^{1 / 2} \rightarrow \infty .
$$

The vorticity field $\omega(x)=\nabla \times \mathbf{v}, x \in R^{3}$, then takes the form

$$
\omega(x)=\omega(r, z) \mathbf{i}_{\theta}, \quad \omega(r, z)=-v_{r}^{z}+v_{z}^{r} .
$$

The flow is governed by the (steady) Euler's equations for an ideal fluid of unit density; namely,

$$
\begin{aligned}
\nabla \cdot \mathbf{v} & =0, \\
\mathbf{v} \cdot \nabla \mathbf{v} & =-\nabla p
\end{aligned}
$$

where the pressure $p(x)$ is also to be determined.

The conservation of mass equation (1.4) (together with axisymmetry) is equivalent to the existence of a stream function $\hat{\psi}(r, z)$ satisfying

$$
v^{r}=-\hat{\psi}_{z} / r, \quad v^{z}=\hat{\psi}_{r} / r .
$$

If we define

$$
\psi=\hat{\psi}+\frac{1}{2} W r^{2}
$$

then condition (1.2) becomes

$$
|\nabla \psi| / r=o(1) \quad \text { as }|x| \rightarrow \infty ;
$$

that is, $\psi(r, z)$ is the stream function for the associated flow which is at rest at infinity. Recalling (1.3) we now have

$$
\omega=-(L \hat{\psi}) / r=-(L \psi) / r
$$

where

$$
L=r \frac{\partial}{\partial r}\left(\frac{1}{r} \frac{\partial}{\partial r}\right)+\frac{\partial^{2}}{\partial z^{2}} .
$$

The conservation of momentum equation (1.5) holds for some $p$ provided

$$
\nabla \times(\mathbf{v} \cdot \nabla \mathbf{v})=0 .
$$

The well-known identity

$$
\mathbf{v} \cdot \nabla \mathbf{v}=\frac{1}{2} \nabla|\mathbf{v}|^{2}+\omega \times \mathbf{v}
$$

yields

$$
\nabla \times(\mathbf{v} \cdot \nabla \mathbf{v})=\nabla \times(\omega \times \mathbf{v})=\mathbf{v} \cdot \nabla \omega-\omega \cdot \nabla \mathbf{v},
$$

since also $\nabla \cdot v=\nabla \cdot \omega=0$. Furthermore,

$$
\mathbf{v} \cdot \nabla \omega=(\mathbf{v} \cdot \nabla \omega) \mathbf{i}_{\theta}, \quad \omega \cdot \nabla \mathbf{v}=\omega\left(v^{r} / r\right) \mathbf{i}_{\theta} .
$$

Therefore, if we let

$$
\zeta(r, z)=\omega(r, z) / r,
$$

then (1.11) reduces to simply

$$
\mathbf{v} \cdot \nabla \zeta=0 .
$$

Recalling (1.6) we see that (1.13) can be written as

$$
\partial(\hat{\psi}, \zeta) / \partial(r, z)=0 .
$$


Equation (1.14) is, in turn, equivalent to the existence of a functional dependence $\Phi$ $(\nabla \Phi \neq 0)$ such that

$$
\Phi(\hat{\psi}, \zeta)=0
$$

In particular, we shall seek solutions for which

$$
\zeta=f(\hat{\psi}-\gamma)
$$

for some (flux) constant $\gamma$; the so-called vorticity function $f(t)$ is assumed to be a nondecreasing function on $(-\infty, \infty)$ with $f(t)=0$ for $t<0, f(t)>0$ for $t>0$.

In summary, a solution of the problem (1.1), (1.2), (1.4) and (1.5) is obtained whenever (1.15) holds for $\psi$ satisfying condition (1.8) and the equation

$$
-(L \psi) / r^{2}=\zeta
$$

Introducing the (adjusted) stream function

$$
u(r, z)=\psi-\frac{1}{2} W r^{2}-\gamma
$$

equations (1.15) and (1.16) combine to give the semilinear elliptic equation

$$
L u+r^{2} f(u)=0 .
$$

Also, the support of $\zeta$ (the vortex core) is characterized by

$$
\operatorname{supp} \zeta=\bar{\Omega}, \quad \Omega=\left\{x \in R^{3} ; u(x)>0\right\} .
$$

For the solutions we shall find $\bar{\Omega}$ is always compact.

In order to pose the problem exclusively in terms of $\zeta$ we require the inverse of the operator $-L / r^{2}$.

LEMMA 1.1. Let

$$
K\left(x, x^{\prime}\right)=r r^{\prime} \cos \left(\theta-\theta^{\prime}\right) / 4 \pi\left|x-x^{\prime}\right|
$$

where $x=(r, \theta, z), x^{\prime}=\left(r^{\prime}, \theta^{\prime}, z^{\prime}\right)$. For $\zeta(r, z)=\zeta(x)$ any bounded, measurable function with compact support in $R^{3}$ let

$$
\psi(r, z)=\psi(x)=\int_{R^{3}} K\left(x, x^{\prime}\right) \zeta\left(x^{\prime}\right) d x^{\prime} .
$$

Then

$$
\begin{aligned}
-(L \psi) / r^{2} & =\zeta \quad \text { a.e., } \\
\psi / r=O\left(|x|^{-2}\right), \quad|\nabla \psi| / r & =O\left(|x|^{-3}\right), \quad \text { as }|x| \rightarrow \infty .
\end{aligned}
$$

Proof. Let $\omega(\mathrm{x})=r \zeta(r, z) \mathbf{i}_{\theta}$. Then

$$
\mathbf{B}(x)=\frac{1}{4 \pi} \int_{R^{3}} \frac{1}{\left|x-x^{\prime}\right|} \omega\left(x^{\prime}\right) d x^{\prime}
$$

satisfies

$$
-\triangle B=\omega \quad \text { a.e. }
$$

By direct calculation,

$$
\int_{-\pi}^{\pi} \frac{1}{\left|x-x^{\prime}\right|} \mathbf{i}_{\theta^{\prime}} d \theta^{\prime}=\left\{\int_{-\pi}^{\pi} \frac{\cos \left(\theta-\theta^{\prime}\right)}{\left|x-x^{\prime}\right|} d \theta^{\prime}\right\} \mathbf{i}_{\theta}
$$


Thus,

$$
\mathbf{B}(x)=\left\{\frac{1}{4 \pi} \int_{R^{3}} \frac{\cos \left(\theta-\theta^{\prime}\right)}{\left|x-x^{\prime}\right|} r^{\prime} \zeta\left(r^{\prime}, z^{\prime}\right) d x^{\prime}\right\} \mathbf{i}_{\theta},
$$

and hence, by virtue of (1.20) and (1.21),

$$
r \mathrm{~B}(x)=\psi(r, z) \mathbf{i}_{\theta} .
$$

Now we compute

$$
\nabla \times \mathbf{B}=\left(-\psi_{z} / r\right) \mathbf{i}_{r}+\left(\psi_{r} / r\right) \mathbf{i}_{z} .
$$

Also, since $\nabla \cdot \mathbf{B}=0$, we have

$$
-\triangle \mathbf{B}=\nabla \times(\nabla \times \mathbf{B})=-(L \psi) / \mathbf{r i}_{\theta} .
$$

Combining this with (1.24) yields the desired result (1.16).

To establish the estimates (1.22) we first note that

$$
\frac{1}{\left|x-x^{\prime}\right|}=\frac{1}{|x|}+\frac{a\left(x, x^{\prime}\right)}{|x|^{2}}, \quad \nabla_{x} \frac{1}{\left|x-x^{\prime}\right|}=\nabla_{x} \frac{1}{|x|}+\frac{\mathbf{A}\left(x, x^{\prime}\right)}{|x|^{3}}
$$

for certain functions $a, \mathbf{A}=O(1)$ as $|x| \rightarrow \infty, x^{\prime} \in \operatorname{supp} \zeta$. Now using the cancellation property

$$
\int_{R^{3}} r^{\prime} \zeta\left(r^{\prime}, z^{\prime}\right) \mathbf{i}_{\theta^{\prime}} d x^{\prime}=0
$$

we find that

$$
\begin{gathered}
\mathbf{B}(x)=\frac{1}{4 \pi}|x|^{-2} \int_{R^{3}} a\left(x, x^{\prime}\right) \omega\left(x^{\prime}\right) d x^{\prime}, \\
\nabla \times \mathbf{B}(x)=\frac{1}{4 \pi}|x|^{-3} \int_{R^{3}} \mathbf{A}\left(x, x^{\prime}\right) \times \omega\left(x^{\prime}\right) d x^{\prime} .
\end{gathered}
$$

Recalling (1.25) and (1.26), the estimates (1.22) now follow.

The Green's function for the operator $-L / r^{2}$ on the half-plane $H$ with measure $r d r d z$ is clearly given by

$$
\begin{aligned}
G\left(r, z, r^{\prime}, z^{\prime}\right) & =\int_{-\pi}^{\pi} K\left(x, x^{\prime}\right) d \theta^{\prime} \quad(\theta=0) \\
& =\frac{r r^{\prime}}{4 \pi} \int_{-\pi}^{\pi} \frac{\cos \theta^{\prime} d \theta^{\prime}}{\left[\left(z-z^{\prime}\right)^{2}+r^{2}+r^{\prime 2}-2 r r^{\prime} \cos \theta^{\prime}\right]^{1 / 2}},
\end{aligned}
$$

since then (1.21) takes the form

$$
\psi(r, z)=\iint_{H} G\left(r, z, r^{\prime}, z^{\prime}\right) \zeta\left(r^{\prime}, z^{\prime}\right) r^{\prime} d r^{\prime} d z^{\prime} .
$$

Using Lemma 1.1 we have the following expressions for the total kinetic energy of the flow:

$$
\begin{aligned}
E & =\frac{1}{2} \int_{R^{3}} \frac{1}{r^{2}}|\nabla \psi|^{2} d x=\frac{1}{2} \int_{R^{3}} \psi \zeta d x \\
& =\frac{1}{2} \int_{R^{3}} \int_{R^{3}} K\left(x, x^{\prime}\right) \zeta(x) \zeta\left(x^{\prime}\right) d x d x^{\prime},
\end{aligned}
$$

where the second equality follows from integration by parts. 
The total impulse required to generate the flow from rest is defined by (see [3])

$$
\mathbf{P}=\frac{1}{2} \int_{R^{3}} \mathbf{x} \times \omega(x) d x .
$$

This takes the form

$$
\mathbf{P}=P \mathbf{i}_{z}, \quad P=\frac{1}{2} \int_{R^{3}} r^{2} \zeta(x) d x
$$

provided that

$$
\int_{R^{3}} r z \zeta(x) d x=0
$$

In the sequel we shall assume that $\zeta(r, z)=\zeta(r,-z)$ holds; hence, (1.32) is assured.

In posing the steady vortex ring problem variationally we shall maximize the energy $E(\zeta)$ over a certain class of functions $\zeta$ subject to the constraint that the impulse $P$ be prescribed.

2. Existence theorems. Let $\mathcal{Q}_{\lambda}$ denote the class of measurable functions $\zeta \geqslant 0$ a.e. on $R^{3}$ satisfying the following conditions:

$$
\begin{gathered}
\zeta(x)=\zeta(r, z)=\zeta(r,-z), \\
\frac{1}{2} \int_{R^{3}} r^{2} \zeta(x) d x=1, \\
\int_{R^{3}} \zeta(x) d x \leqslant 1, \\
\underset{x \in R^{3}}{\operatorname{ess} \sup _{x}} \zeta(x) \leqslant \lambda, \quad 0<\lambda<\infty .
\end{gathered}
$$

Let $\mathbb{Q}_{\infty}$ denote the (larger) class for which condition (2.4) is removed.

The energy functional $E(\zeta)$ is defined on the class $\mathbb{Q}_{\lambda}(0<\lambda<\infty)$ by

$$
E(\zeta)=\frac{1}{2} \int_{R^{3}} \int_{R^{3}} K\left(x, x^{\prime}\right) \zeta(x) \zeta\left(x^{\prime}\right) d x d x^{\prime},
$$

where $K\left(x, x^{\prime}\right)$ is given by (1.20). We consider the variational problem to determine $\zeta \in \mathbb{Q}_{\lambda}$ such that

$$
E(\zeta)=\max _{\tilde{\zeta} \in \mathbb{Q}_{\lambda}} E(\tilde{\zeta})
$$

A solution of this problem is provided by the following theorem whose proof is given in $\$ 5$.

THEOREM 2.1. There exists $\zeta \in \mathbb{Q}_{\lambda}$ such that (2.6) holds. Furthermore, there exist constants $W>0, \gamma \geqslant 0$ such that

$$
\zeta=\lambda I_{\Omega} \text { a.e. }
$$

where

$$
\Omega=\left\{x \in R^{3} ; u(x) \equiv \psi(x)-\frac{1}{2} W r^{2}-\gamma>0\right\}
$$


with $\psi$ defined by (1.21); $\Omega$ is a bounded, open subset of $R^{3}$, and

$$
\Omega=\{x=(r, \theta, z) ;|z|<Z(r)\}
$$

for some function $Z(r) \geqslant 0$.

For each value of the free parameter $\lambda$ the solution obtained represents a steady vortex ring corresponding to the vorticity function

$$
f(t)=\lambda I_{\{t>0\}} \text {. }
$$

The normalizations taken in (2.2)-(2.4) can always be achieved by a similarity transformation. Let $\mathbb{Q}_{P, \Gamma, \Lambda}$ denote the class of $\zeta \geqslant 0$ satisfying (2.1) and the constraints

$$
\begin{gathered}
\frac{1}{2} \int_{R^{3}} r^{2} \zeta(x) d x=P \quad \text { (total impulse), } \\
\int_{R^{3}} \zeta(x) d x \leqslant \Gamma \quad \text { (total circulation) } \times 2 \pi, \\
\underset{x \in R^{3}}{\text { ess sup }} \zeta(x) \leqslant \Lambda \quad \text { (vortex strength) }
\end{gathered}
$$

for prescribed positive constants $P, \Gamma, \Lambda$. We consider the general problem to determine $\zeta \in \mathbb{Q}_{P, \Gamma, \Lambda}$ such that

$$
E(\zeta)=\max _{\tilde{\zeta} \in \mathbb{Q}_{P, \Gamma, \Lambda}} E(\tilde{\zeta}) .
$$

Making the change of variables $x=a \hat{x}, \zeta=b \hat{\zeta}$, and choosing $a=P^{1 / 2} \Gamma^{-1 / 2}$, $b=P^{-3 / 2} \Gamma^{5 / 2}$, the constraints (2.10) and (2.11) become, respectively,

$$
\frac{1}{2} \int_{R^{3}} \hat{r}^{2} \hat{\zeta}(\hat{x}) d \hat{x}=1, \quad \int_{R^{3}} \hat{\zeta}(\hat{x}) d \hat{x}<1 .
$$

Thus problem (2.13) is transformed into problem (2.6) with $\lambda=P^{3 / 2} \Gamma^{-5 / 2} \Lambda$. Other quantities involved transform as

$$
E=P^{1 / 2} \Gamma^{3 / 2} \hat{E}, \quad W=P^{-1 / 2} \Gamma^{3 / 2} \hat{W}, \quad \gamma=P^{1 / 2} \Gamma^{1 / 2} \hat{\gamma}
$$

In the remainder of the paper we shall deal with the normalized problem (2.1)(2.6), since all results immediately transform to the general problem.

We note that unlike (2.4)-which becomes equality for any solution-the constraint (2.3) may be a strict inequality for some solutions. In the variational conditions of Theorem 2.1 the constants $W$ and $\gamma$ arise as Lagrange multipliers for the constraints (2.2) and (2.3), respectively. Thus, if $\gamma>0$ (as is the case for sufficiently large $\lambda$ ) then equality holds in (2.3), while if $\gamma=0$ (as is the case for Hill's spherical vortex) then inequality may hold; for more details, see Remark 2 at the end of $\$ 5$.

A related family of variational problems is derived from the penalized energy functional

$$
E_{\beta}(\zeta)=E(\zeta)-\beta \lambda \int_{R^{3}}\left[\frac{\zeta(x)}{\lambda}\right]^{1+1 / \beta} d x
$$


defined on the class

$$
\mathbb{Q}_{\infty, \beta}=\mathbb{Q}_{\infty} \cap L^{1+1 / \beta}\left(R^{3}\right) \quad(0<\beta<\infty) .
$$

We consider the (penalized) problem to determine $\zeta \in \mathbb{Q}_{\infty, \beta}$ such that

$$
E_{\beta}(\zeta)=\max _{\tilde{\zeta} \in \mathbb{Q}_{\infty, \beta}} E_{\beta}(\tilde{\zeta})
$$

THEOREM 2.2. There exists $\zeta \in \mathbb{Q}_{\infty, \beta}$ such that (2.16) holds for any prescribed $0<\lambda<\infty$, provided $0<\beta<5$. Furthermore, there exist constants $W>0, \gamma>0$ such that

$$
\zeta=\lambda\left(u^{+} /(1+\beta)\right)^{\beta} \text { a.e. }
$$

where

$$
u(x) \equiv \psi(x)-\frac{1}{2} W r^{2}-\gamma
$$

with $\psi$ defined by (1.21); $\zeta$ has compact support in $R^{3}$, and $\zeta(r, z)$ is nonincreasing as a function of $z$ for $z>0$.

Clearly, these solutions of the vortex ring problem correspond to a vorticity function of the form

$$
f(t)= \begin{cases}\lambda(t /(1+\beta))^{\beta}, & t>0 \\ 0, & t<0 .\end{cases}
$$

It is evident that the variational equation (2.17) tends, as $\beta \rightarrow 0$, to (2.7). In fact, Theorem 2.1 is proved by first obtaining the solutions asserted by Theorem 2.2 and then taking the limit of these solutions over some sequence $\beta_{j} \rightarrow 0$; the sequence of solutions converges weakly in $L^{p}\left(R^{3}\right)$ for every $1<p<\infty$. Theorem 2.2 itself is proved in $\$ 4$.

REMARK 1. There is a striking analogy between the steady vortex ring problem and the problem to determine the axisymmetric equilibrium figures of a rotating, self-gravitating fluid mass. Problem (2.6) is analogous to the case of an incompressible fluid, as studied by Auchmuty [1], while problem (2.16) is analogous to the corresponding compressible case, as studied by Auchmuty and Beals [2] and Friedman and Turkington [16]. The variational approach developed for these problems motivates the formulation of problems (2.6) and (2.16) given above. The energy functional for the rotating fluid mass problem-although it involves an additional term to the analog of $E(\zeta)$-is, however, maximized over a class of functions with fewer (and simpler) constraints. The presence of (2.4), in particular, among the constraints for problem (2.6), causes technical difficulties; we overcome the difficulties by obtaining the solution as limits of solutions of the penalized problem (2.16), for which the variational conditions can be derived using standard methods.

REMARK 2. In the variational principle used by Benjamin [4] the energy $E(\zeta)$ is maximized subject to the constraints (2.1) and (2.2) but with (2.3) and (2.4) replaced by the condition that $\zeta$ be a rearrangement of a given function $\zeta_{0}$. By 
prescribing the distribution function of $\zeta$ in this manner the vorticity function $f(t)$-a priori unknown-is determined along with the solution (and the Lagrange multiplier $W$ ).

REMARK 3. The approach of Fraenkel and Berger [13] is to pose a variational problem for the function $u$ defined in (1.17); the propagation speed $W$, the flux constant $\gamma$, and the vorticity function $f(t)$ (suitably normalized) are prescribed in this approach. The method is more complicated technically than a variational principle based on $\zeta$ mainly due to the fact that $u$ does not have compact support. The approach of Fraenkel and Berger is used also in more recent works by Berestycki [5] and Ni [20].

REMARK 4. Using an analysis based on (1.18), Caffarelli and Friedman [9] have proved that the number of components of $\Omega$ is finite, and that the free boundary $\partial \Omega$ (as given by $z=Z(r)$ ) is real analytic. Benjamin [13] asserts that there is just one component, arguing that a positive second variation for $E(\zeta)$ is obtained for (infinitesimal) relative displacements of two distinct components.

3. Preliminary identities and estimates. We shall often use the integration by parts formula

$$
\int_{H} \frac{1}{r^{2}}\left(u_{r} v_{r}+u_{z} v_{z}\right) d z=-\int_{H} \frac{1}{r^{2}} u L v d x
$$

for functions $u(r, z), v(r, z)$ either of compact support or vanishing sufficiently fast as $r^{2}+z^{2} \rightarrow \infty$; recall that $L$ is defined in (1.10). In particular, the calculation (1.29) expressing $E$ in terms of $\zeta$ is justified using (3.1) and the estimates for the corresponding $\psi$ given in Lemma 1.1 , provided $\zeta$ has compact support.

We now give another formula for $E$.

LEMMA 3.1. Let $\zeta$ be any bounded, measurable function on $H$ for which

$$
E(\zeta)=\frac{1}{2} \int_{H} \frac{1}{r^{2}}\left(\psi_{r}^{2}+\psi_{z}^{2}\right) d x<\infty
$$

Then

$$
E(\zeta)=\int_{H}\left(r \psi_{r}+z \psi_{z}\right) \zeta d x
$$

Proof. Set $\sigma=\frac{1}{2}\left(r^{2}+z^{2}\right), \eta=\frac{1}{2}\left(\psi_{r}^{2}+\psi_{z}^{2}\right)$. Since $L \sigma=1$, we have

$$
E(\zeta)=\int_{H} \frac{1}{r^{2}} \eta L \sigma d x=-\int_{H} \frac{1}{r^{2}}\left(\sigma_{r} \eta_{r}+\sigma_{z} \eta_{z}\right) d x=-\int_{H} \frac{1}{r^{2}}\left(r \eta_{r}+z \eta_{z}\right) d x
$$

where (3.1) has been used (the justification will be given below). Expanding the integrand we find that

$$
r \eta_{r}+z \eta_{z}=\psi_{r}\left(r \psi_{r}+z \psi_{z}\right)_{r}+\psi_{z}\left(r \psi_{r}+z \psi_{z}\right)_{z}-\left(\psi_{r}^{2}+\psi_{z}^{2}\right)
$$

Putting this into (3.4) we get

$$
E(\zeta)=-\int_{H} \frac{1}{r^{2}}\left[\psi_{r}\left(r \psi_{r}+z \psi_{z}\right)_{r}+\psi_{z}\left(r \psi_{r}+z \psi_{z}\right)_{z}\right] d x+2 E(\zeta)
$$


so

$$
\begin{aligned}
E(\zeta) & =\int_{H} \frac{1}{r^{2}}\left[\psi_{r}\left(r \psi_{r}+z \psi_{z}\right)_{r}+\psi_{z}\left(r \psi_{r}+z \psi_{z}\right)_{z}\right] d x \\
& =-\int_{H} \frac{1}{r^{2}}\left(r \psi_{r}+z \psi_{z}\right) L \psi d x,
\end{aligned}
$$

which yields (3.3).

We must, however, rigorously justify the use of formula (3.1) in the derivations (3.4) and (3.5) above. If the integration by parts is carried out instead on the finite domain

$$
D_{a}=\{(r, z) \in H ; 0 \leqslant r<a,|z|<a\},
$$

then it is easily seen that the boundary integrals arising (in both cases) tend to zero as $a \rightarrow \infty$ provided $\int_{\partial D_{a}} r^{-1} \eta d s=o(1 / a)$ as $a \rightarrow \infty$. The latter condition is assured for some sequence $a=a_{n} \rightarrow \infty$ by the hypothesis (3.2), since it means that

$$
\int_{0}^{\infty} \int_{-\infty}^{\infty} \frac{1}{r} \eta d r d z<\infty
$$

This completes the proof.

RemarK. An equivalent form of (3.3) is discussed in Batchelor [3, p. 520].

We now relate the energy $E(\zeta)$ to a functional introduced by Fraenkel and Berger [13].

For a given vorticity function $f(t)$ let

$$
F(t)=\int_{0}^{t} f\left(t^{\prime}\right) d t^{\prime}
$$

then

$$
F(t)= \begin{cases}\lambda t^{+} & \text {when (2.9) holds, } \\ \lambda\left(t^{+} /(1+\beta)\right)^{1+\beta} & \text { when (2.19) holds }\end{cases}
$$

Let

$$
J(u)=\int_{H} F(u) d x
$$

recall that $u(r, z)$ is defined in (1.17). Several other expressions for $J(u)$ can be given; namely,

$$
(1+\beta) J(u)=\int_{H} u \zeta d x=-\int_{H} \frac{1}{r^{2}} u L u d x=\int_{\Omega} \frac{1}{r^{2}}\left(u_{r}^{2}+u_{z}^{2}\right) d x,
$$

where $\Omega=\left\{x \in R^{3} ; u(x)>0\right\}$. Thus, $\frac{1}{2}(1+\beta) J(u)$ represents the kinetic energy of the steady flow (with stream function $u$ ) confined to the vortex core $\Omega$.

We note that when (2.9) holds the above formulas are valid if formally we set $\beta=0$.

LEMMA 3.2. Let $\zeta$ be a solution of either (2.6) or (2.16) with compact support. Then

$$
E(\zeta)=-3 J(u)+2 W=\frac{1}{2}[(1+\beta) J(u)+W+\gamma] .
$$

The first identity is briefly mentioned in Fraenkel and Berger [13, p. 42]. 
Proof. The assertion that $E(\zeta)$ equals the right-hand side of $(3.10)$ is immediate from

$$
\int_{H} u \zeta d x-\int_{H} \psi \zeta d x-\frac{W}{2} \int_{H} r^{2} \zeta d x-\gamma \int_{H} \zeta d x=2 E-W-\gamma
$$

The first identity in (3.10) follows from (3.3); noting that $u_{r}=\psi_{r}-W r, u_{z}=\psi_{z}$ we have

$$
E(\zeta)=\int_{H}\left(r u_{r}+z u_{z}\right) \zeta d x+\int_{H} r(W r) \zeta d x
$$

Integrating by parts we find

and, similarly,

$$
\begin{aligned}
\int_{H} r u_{r} \zeta d x & =\int_{H} r F(u)_{r} d x=2 \pi \iint_{H} r^{2} F(u)_{r} d r d z \\
& =-4 \pi \int_{H} r F(u) d r d z=-2 \int_{H} F(u) d x
\end{aligned}
$$

$$
\int_{H} z u_{z} \zeta d x=-\int_{H} F(u) d x
$$

Thus, (3.11) becomes

$$
E(\zeta)=-3 \int_{H} F(u) d x+W \int_{H} r^{2} \zeta d x=-3 J(u)+2 W .
$$

REMARK. It is important for later application that we observe that (3.10) remains valid for solutions of (2.16) without the assumption of compact support. Indeed, if the integration by parts in the proof of Lemma 3.2 is carried out on the finite domain $D_{a}$ (defined in (3.6)) then the boundary integrals which arise are of the form

$$
\int_{r=a} r^{2} F(u) d z, \int_{z= \pm a} r z F(u) d r
$$

Noting that $F(u)=\lambda[\zeta(x) / \lambda]^{1+1 / \beta}$ we see that there is a sequence $a=a_{n} \rightarrow \infty$ for which these integrals tend to zero since the condition

implies that

$$
\iint_{H} r \zeta^{1+1 / \beta} d r d z<\infty
$$

$$
\int_{-\infty}^{\infty} r^{2} \zeta^{1+1 / \beta} d z, \quad \int_{0}^{\infty} r z \zeta^{1+1 / \beta} d r=o(1)
$$

for $r=a_{n},|z|=a_{n}$, respectively.

We now turn our attention to certain "potential-type" estimates for $\psi$, as given by (1.28).

LEMMA 3.3. Let $s=\left[\left(r-r^{\prime}\right)^{2}+\left(z-z^{\prime}\right)^{2}\right]^{1 / 2}$. There holds

$$
G\left(r, z, r^{\prime}, z^{\prime}\right) \leqslant \begin{cases}C r \log (r / s), & s<r / 2 \\ C r^{2} r^{\prime 2} / s^{3}, & s>r / 2\end{cases}
$$

where $C$ is a (sufficiently large) absolute positive constant. 
Proof. Recall that $G$ is defined by (1.27). Letting

$$
\xi^{2}=\left[\left(z-z^{\prime}\right)^{2}+\left(r-r^{\prime}\right)^{2}\right] / 4 r r^{\prime} \quad(\xi>0)
$$

we first show that

$$
\begin{gathered}
G\left(r, z, r^{\prime}, z^{\prime}\right) \leqslant C\left(r r^{\prime}\right)^{1 / 2} \log (C / \xi) \quad \text { if } \xi \leqslant 1, \\
G\left(r, z, r^{\prime}, z^{\prime}\right) \leqslant C\left(r r^{\prime}\right)^{1 / 2} \xi^{-3} \quad \text { if } \xi \geqslant 1 .
\end{gathered}
$$

Estimate (3.13) is derived from the formula

$$
G\left(r, z, r^{\prime}, z^{\prime}\right)=\left[\left(r r^{\prime}\right)^{1 / 2} / 2 \pi\right]\{(2 / k-k) K(k)-(2 / k) E(k)\},
$$

where $K$ and $E$ are the complete elliptic integrals of the first and second kind [8, formulas 291.00 and 291.01 ] and

$$
k^{2}=\frac{4 r r^{\prime}}{\left(z-z^{\prime}\right)^{2}+\left(r+r^{\prime}\right)^{2}}, \quad k^{\prime 2}=\frac{\left(z-z^{\prime}\right)^{2}+\left(r-r^{\prime}\right)^{2}}{\left(z-z^{\prime}\right)^{2}+\left(r+r^{\prime}\right)^{2}}, \quad k^{2}+k^{\prime 2}=1 .
$$

Since $\xi \leqslant 1$ implies that $k^{\prime 2} \leqslant \frac{1}{2}$ we apply the asymptotic formulas

$$
K(k)=\log \left(4 / k^{\prime}\right)+o(1), \quad E(k)=1+o(1) \quad\left(\text { as } k^{\prime} \rightarrow 0\right)
$$

to (3.15); then (3.13) follows directly. Estimate (3.14) follows from the expansion

$$
\left[\left(z-z^{\prime}\right)^{2}+r^{2}+r^{\prime 2}-2 r r^{\prime} \cos \theta^{\prime}\right]^{-1 / 2}=\left(4 r r^{\prime}\right)^{-1 / 2}\left\{\xi^{-1}+B\left(\xi, \theta^{\prime}\right) \xi^{-3}\right\}
$$

where $\left|B\left(\xi, \theta^{\prime}\right)\right| \leqslant C$ for $\xi \geqslant 1,-\pi \leqslant \theta^{\prime} \leqslant \pi$. Applying this to (1.27) and noting the cancellation of the term involving $\xi^{-1},(3.14)$ follows. The required estimate (3.12) follows from (3.13) and (3.14) in an entirely straightforward manner.

In the following lemmas we assume that $\zeta \in \mathbb{Q}_{\infty, \beta}$, and we let $N=N(\zeta)$ denote the norm

$$
N=\left\{\int_{H} \zeta^{1+1 / \beta} d x\right\}^{\beta /(1+\beta)} \quad(0<\beta<\infty)
$$

LEMMA 3.4. Assume that $\zeta(r, z)$ is a nonincreasing function of $z$ for $z>0$. Then for $0<\beta \leqslant \beta^{*}$ there holds

$$
\psi(r, z) \leqslant \psi(r, 0) \leqslant C(N+1) \min \left\{r, r^{-1+\varepsilon}\right\} \quad(0<\varepsilon<1) ;
$$

the constant $C$ depends only on $\beta^{*}, \varepsilon$.

Proof. It is clear that $\psi(r, 0)=\max _{z} \psi(r, z)$. We write $\psi(r, 0)=\psi_{1}(r, 0)+$ $\psi_{2}(r, 0)$ where

$$
\begin{aligned}
\psi_{1}(r, 0) & =\iint_{s<r / 2} G\left(r, 0, r^{\prime}, z^{\prime}\right) \zeta\left(r^{\prime}, z^{\prime}\right) r^{\prime} d r^{\prime} d z^{\prime} \\
& \leqslant C r \int_{s<r / 2} \log \frac{r}{s} \zeta\left(r^{\prime}, z^{\prime}\right) r^{\prime} d r^{\prime} d z^{\prime}, \\
\psi_{2}(r, 0) & =\iint_{s>r / 2} G\left(r, 0, r^{\prime}, z^{\prime}\right) \zeta\left(r^{\prime}, z^{\prime}\right) r^{\prime} d r^{\prime} d z^{\prime} \\
& \leqslant C r^{2} \int_{s>r / 2} \frac{r^{\prime 2}}{s^{3}} \zeta\left(r^{\prime}, z^{\prime}\right) r^{\prime} d r^{\prime} d z^{\prime} .
\end{aligned}
$$


Estimation of $\psi_{2}(r, 0)$ : we note that

$$
r^{\prime 2} / s^{3}<C / r \quad \text { whenever } s>r / 2 \text {. }
$$

Thus, recalling (2.3),

$$
\psi_{2}(r, 0) \leqslant C r^{2} \int_{s>r / 2} \frac{C}{r} \zeta\left(r^{\prime}, z^{\prime}\right) r^{\prime} d r^{\prime} d z^{\prime}<C r .
$$

Also, recalling (2.2),

$$
\psi_{2}(r, 0) \leqslant C r^{2} \int_{s>r / 2} \frac{r^{2}}{(r / 2)^{3}} \zeta\left(r^{\prime}, z^{\prime}\right) r^{\prime} d r^{\prime} d z^{\prime}<C r^{-1}
$$

Hence,

$$
\psi_{2}(r, 0)<C \min \left\{r, r^{-1}\right\} .
$$

Estimation of $\psi_{1}(r, 0)$ : for any $0<\alpha<\infty$ we have, by Hölder's inequality,

$$
\begin{aligned}
\int_{s<r / 2} \log \frac{r}{s} \zeta\left(r^{\prime}, z^{\prime}\right) r^{\prime} d r^{\prime} d z^{\prime}< & \left\{\iint_{s<r / 2}\left(\log \frac{r}{s}\right)^{1+\alpha} r^{\prime} d r^{\prime} d z^{\prime}\right\}^{1 /(1+\alpha)} \\
& \cdot\left\{\iint_{s<r / 2} \zeta^{1+1 / \alpha} r^{\prime} d r^{\prime} d z^{\prime}\right\}^{\alpha /(1+\alpha)} .
\end{aligned}
$$

But clearly

$$
\int_{s<r / 2}\left(\log \frac{r}{s}\right)^{1+\alpha} r^{\prime} d r^{\prime} d z^{\prime}<C r \int_{0}^{r / 2}\left(\log \frac{r}{s}\right)^{1+\alpha} s d s=C_{\alpha} r^{3}
$$

Thus,

$$
\psi_{1}(r, 0) \leqslant C_{\alpha} r^{1+\delta}\|\zeta\|_{L^{1+1 / \alpha(s<r / 2)}} \quad(\delta=3 /(1+\alpha)) .
$$

We now apply the standard interpolation inequality

$$
\|\zeta\|_{L^{1+1 / \alpha}} \leqslant\|\zeta\|_{L^{1}-a}^{1-\zeta \|_{L^{1+1 / \beta}}^{a}} \quad(\beta<\alpha<\infty)
$$

where $a=(1+\beta) /(1+\alpha)(0<a<1)$; each $L^{p}$ norm is taken with respect to the measure $r d r d z$ on the set $\{(r, z) \in H ; s<r / 2\}$. Since, by (2.2) and (2.3),

$$
\|\zeta\|_{L^{1}(s<r / 2)}<C \min \left\{1, r^{-2}\right\},
$$

we conclude from (3.24) that

$$
\|\zeta\|_{L^{1+1 / \alpha(s<r / 2)}}<C N^{a} \min \left\{1, r^{-2(1-a)}\right\} .
$$

Thus,

$$
\psi_{1}(r, 0)<C_{\alpha} N^{a} r^{1+\delta} \min \left\{1, r^{-2(1-a)}\right\} .
$$

For $r<1$ we take simply $\alpha=\beta$, so that

$$
\psi_{1}(r, 0)<C_{\beta^{*}}(N+1) r \quad\left(0<\beta<\beta^{*}\right) .
$$

For $r>1$ we take $\alpha$ sufficiently large (depending on $\beta^{*}, \varepsilon$ ) so that

$$
\psi_{1}(r, 0) \leqslant C_{\beta^{*}, \varepsilon}(N+1) r^{-1+e} \quad\left(0<\beta \leqslant \beta^{*}\right)
$$


specifically we choose $\alpha$ so that $\left(5+3 \beta^{*}\right) /(1+\alpha)<\varepsilon$. Together these estimates yield

$$
\psi_{1}(r, 0) \leqslant C_{\beta^{*}, \varepsilon}(N+1) \min \left\{r, r^{-1+\varepsilon}\right\} .
$$

Now combining (3.22) and (3.25) we obtain the statement of the lemma.

LEMMA 3.5. Assume that $\zeta(r, z)$ is a nonincreasing function of $z$ for $z>0$. Then for $0<\beta \leqslant \beta^{*}$ there holds

$$
\psi(r, z) \leqslant C(N+1) A^{-1+\varepsilon} \min \left\{r, r^{-1+\varepsilon}\right\}+C r^{2}(A / z)^{3}
$$

provided $r / 2<z / A$ and $A>1$; the constant $C$ depends only on $\beta^{*}, \varepsilon$.

Proof. The monotonicity of $\zeta(r, z)$ in $z$ implies that, for any $A>1$,

$$
\begin{gathered}
\iint_{\left|z^{\prime}-z\right|<z / A} \zeta\left(r^{\prime}, z^{\prime}\right) r^{\prime} d r^{\prime} d z^{\prime} \leqslant \frac{1}{A} \iint_{z^{\prime}>0} \zeta\left(r^{\prime}, z^{\prime}\right) r^{\prime} d r^{\prime} d z^{\prime}, \\
\iint_{\left|z^{\prime}-z\right|<z / A} r^{\prime 2} \zeta\left(r^{\prime}, z^{\prime}\right) r^{\prime} d r^{\prime} d z^{\prime} \leqslant \frac{1}{A} \iint_{z^{\prime}>0} r^{\prime 2} \zeta\left(r^{\prime}, z^{\prime}\right) r^{\prime} d r^{\prime} d z^{\prime} .
\end{gathered}
$$

Using these facts we can modify the proof of the previous lemma for large $z$. As in the preceding proof we write $\psi(r, z)=\psi_{1}(r, z)+\psi_{2}(r, z)$. Estimation of $\psi_{2}(r, z)$ :

$$
\begin{aligned}
& \psi_{2}(r, z)=\psi_{2}^{\prime}(r, z)+\psi_{2}^{\prime \prime}(r, z), \\
& \psi_{2}^{\prime}(r, z) \leqslant C r^{2} \int_{\substack{s>r / 2 \\
\left|z-z^{\prime}\right|<z / A}} \frac{r^{\prime 2}}{s^{3}} \zeta\left(r^{\prime}, z^{\prime}\right) r^{\prime} d r^{\prime} d z^{\prime} \leqslant \frac{C}{A} \min \left\{r, r^{-1}\right\} . \\
& \psi_{2}^{\prime \prime}(r, z) \leqslant C r^{2} \int_{\substack{s>r / 2 \\
\left|z-z^{\prime}\right|>z / A}} \frac{r^{\prime 2}}{s^{3}} \zeta\left(r^{\prime}, z^{\prime}\right) r^{\prime} d r^{\prime} d z^{\prime} \leqslant C r^{2}\left(\frac{A}{z}\right)^{3} .
\end{aligned}
$$

Thus,

$$
\psi_{2}(r, z) \leqslant(C / A) \min \left\{r, r^{-1}\right\}+C r^{2}(A / z)^{3} .
$$

Estimation of $\psi_{1}(r, z)$ : we follow the reasoning of the previous proof except that now (noting that $s \leqslant r / 2$ implies $\left|z-z^{\prime}\right|<z / A$ ) we have

$$
\|\zeta\|_{L^{1}(s<r / 2)} \leqslant(C / A) \min \left\{1, r^{-2}\right\} .
$$

Thus, as before,

$$
\psi_{1}(r, z) \leqslant C_{\alpha} N^{a} A^{-1+\varepsilon} r^{1+\delta} \min \left\{1, r^{-2(1-a)}\right\},
$$

with $\delta=3 /(1+\alpha), a=(1+\beta) /(1+\alpha), \beta \leqslant \alpha<\infty$.

Now the proof is completed just as in the preceding proof.

4. Proof of Theorem 2.2. Let $\mathbb{Q}_{\infty, \beta}^{\prime}$ denote the class of nonnegative functions $\zeta \in L^{1+1 / \beta}\left(R^{3}\right)$ satisfying (2.1), (2.3) and (rather than (2.2))

$$
\frac{1}{2} \int_{R^{3}} r^{2} \zeta(x) d x<1
$$

clearly, $\mathbb{Q}_{\infty, \beta} \subseteq \mathbb{Q}_{\infty, \beta}^{\prime}$. Consider the problem:

$$
E_{\beta}(\zeta)=\max _{\tilde{\zeta} \in \mathbb{Q}_{\infty, \beta}^{\prime}} E_{\beta}(\tilde{\zeta})
$$


In order to solve problem (2.16) we first obtain a solution of $\left(2.16^{\prime}\right)$; this is necessary because the possible solutions are not known to have bounded support a priori.

LEMMA 4.1. There exists $\zeta \in \mathbb{Q}_{\infty, \beta}^{\prime}$ such that (2.16') holds for any prescribed $0<\lambda<\infty ; \zeta(r, z)$ is a nonincreasing function of $z$ for $z>0$.

Proof. In what follows we shall assume that $0<\beta<\beta^{*}$ for some fixed $\beta^{*}$ (and the dependence of any constants upon $\beta^{*}$ will not be specified). For any $\zeta \in \mathbb{Q}_{\infty, \beta}^{\prime}$, estimate (3.19) implies that

$$
\sup _{x \in R^{3}} \psi(x) \leqslant C[N(\zeta)+1]
$$

and hence

$$
E(\zeta)=\frac{1}{2} \int_{R^{3}} \psi \zeta d x \leqslant C[N(\zeta)+1]
$$

here we use the fact that the hypothesis of Lemma 3.4 is satisfied by $\zeta^{*}(r, z)$, the symmetrical rearrangement of $\zeta(r, z)$ in the $z$ variable, and $E\left(\zeta^{*}\right)>E(\zeta)$ while $N\left(\zeta^{*}\right)=N(\zeta)$. Furthermore,

$$
\frac{N(\zeta)}{\lambda}=\left\|\frac{\zeta}{\lambda}\right\|_{L^{1+1 / \beta}\left(R^{3}\right)} \leqslant C_{\varepsilon}+\varepsilon \beta \int_{R^{3}}\left(\frac{\zeta}{\lambda}\right)^{1+1 / \beta} d x \quad(\varepsilon>0)
$$

this follows from the elementary inequality

$$
X \leqslant C_{\varepsilon}+\varepsilon \beta X^{1+1 / \beta} \quad(\varepsilon>0),
$$

valid for $0<X<\infty$. Applying (4.4) to estimate the energy in (4.3) we get, fixing $\varepsilon$ sufficiently small,

$$
E(\zeta) \leqslant C_{\lambda}+\frac{\beta \lambda}{2} \int_{R^{3}}\left(\frac{\zeta}{\lambda}\right)^{1+1 / \beta} d x
$$

We now conclude (recall definition (2.14))

$$
E_{\beta}(\zeta) \leqslant C_{\lambda} \text { for all } \zeta \in \mathbb{Q}_{\infty, \beta} \text {. }
$$

Let $\zeta_{j} \in \mathbb{Q}_{\infty, \beta}^{\prime}$ be a maximizing sequence for $E_{\beta}$; that is, $E_{\beta}\left(\zeta_{j}\right)<E_{\beta}\left(\zeta_{j+1}\right)$ and

$$
\lim _{j \rightarrow \infty} E_{\beta}\left(\zeta_{j}\right)=\sup _{\tilde{\zeta} \in \mathbb{Q}_{\infty, \beta}} E_{\beta}(\tilde{\zeta})
$$

It is easy to see that (4.4) and (4.6) imply

$$
N\left(\zeta_{j}\right) \leqslant C_{\lambda}+\left|E_{\beta}\left(\zeta_{1}\right)\right| \quad(j>1) .
$$

Thus we may assume, by extracting a subsequence (and reindexing), that

$$
\zeta_{j} \rightarrow \zeta \text { weakly in } L^{1+1 / \beta}\left(R^{3}\right) \text {. }
$$

The limit $\zeta$ is then an element of $\mathbb{Q}_{\infty, \beta}^{\prime}$ (although not necessarily $\mathbb{Q}_{\infty, \beta}$ ). Also by replacing each $\zeta_{j}(r, z)$ by its symmetrical rearrangement in $z$ (which cannot decrease $E_{\beta}\left(\zeta_{j}\right)$ ) we may assume that each $\zeta_{j}(r, z)$, and hence also $\zeta(r, z)$, is a nonincreasing function of $z$ for $z>0$. 
We shall establish the claim

$$
\lim _{j \rightarrow \infty} E\left(\zeta_{j}\right)=E(\zeta)
$$

From this, combined with the fact that

$$
N(\zeta) \leqslant \lim _{j \rightarrow \infty} N\left(\zeta_{j}\right)
$$

we conclude that

$$
E_{\beta}(\zeta)=\sup _{\zeta \in \mathbb{Q}_{\infty, \beta}} E_{\beta}(\zeta)
$$

as required.

To prove the claim (4.10) we first note that for arbitrary $R>1, A>1$,

$$
\begin{gathered}
\int_{r>R} \psi \zeta d x \leqslant C[N(\zeta)+1] \int_{r>R} r^{-1+\varepsilon \zeta d x} \leqslant C[N(\zeta)+1] R^{-3+e} \\
\int_{\substack{0<r<R \\
|z|>A R}} \psi \zeta d x \leqslant C[N(\zeta)+1] A^{-1+\varepsilon} \int_{R^{3}} \zeta d x+C R^{-3} \int_{R^{3}} r^{2 \zeta} d x \\
\leqslant C[N(\zeta)+1] A^{-1+\varepsilon}+C R^{-3}
\end{gathered}
$$

for $0<\varepsilon<1$ fixed; (4.11) follows by Lemma 3.4 while (4.12) follows by Lemma 3.5. Clearly, estimates analogous to (4.11) and (4.12) hold with $\zeta$ replaced by $\zeta_{j}$. Thus, as $R$ and $A$ may be taken arbitrarily large, it suffices to show that

$$
\int_{\substack{0<r<R \\|z|<A R}} \psi_{j} \zeta_{j} d x \rightarrow \int_{\substack{0<r<R \\|z|<A R}} \psi \zeta d x \quad \text { as } j \rightarrow \infty
$$

$\psi_{j}$ being defined as usual corresponding to $\zeta_{j}$. Also, recalling Lemma 3.3, for $0<r \leqslant R,|z| \leqslant A R$,

$$
\iint_{s>R} G\left(r, z, r^{\prime}, z^{\prime}\right) \zeta\left(r^{\prime}, z^{\prime}\right) r^{\prime} d r^{\prime} d z^{\prime} \leqslant C R^{-3} r^{2}
$$

and hence

$$
\iint_{\substack{0<r<R \\|z|<A R}} \int_{s>R} G\left(r, z, r^{\prime}, z^{\prime}\right) \zeta(r, z) \zeta\left(r^{\prime}, z^{\prime}\right) r d r d z r^{\prime} d r^{\prime} d z^{\prime} \leqslant C R^{-3} ;
$$

also the analogous estimate holds for $\zeta_{j}$. In view of this it suffices to show that

$$
\begin{aligned}
& \iint_{D} \iint_{D} G\left(r, z, r^{\prime}, z^{\prime}\right) \zeta_{j}(r, z) \zeta_{j}\left(r^{\prime}, z^{\prime}\right) r d r d z r^{\prime} d r^{\prime} d z^{\prime} \\
& \rightarrow \iint_{D} \iint_{D} G\left(r, z, r^{\prime}, z^{\prime}\right) \zeta(r, z) \zeta\left(r^{\prime}, z^{\prime}\right) r d r d z r^{\prime} d r^{\prime} d z^{\prime}
\end{aligned}
$$

where $D=\{(r, z) \in H ; 0 \leqslant r \leqslant 2 R,|z| \leqslant(A+1) R\}$ is a bounded domain. But now the result follows by standard arguments since $G \in L^{1+\beta}(D \times D)$ and $\zeta_{j}(r, z) \zeta_{j}\left(r^{\prime}, z^{\prime}\right) \rightarrow \zeta(r, z) \zeta\left(r^{\prime}, z^{\prime}\right)$ weakly in $L^{1+1 / \beta}(D \times D)$ (in the product measure $\left.r d r d z r^{\prime} d r^{\prime} d z^{\prime}\right)$. This completes the proof of the claim and also the lemma.

The positivity of $E_{\beta}(\zeta)$ is crucial for several of the subsequent steps. 
LEMMA 4.2. (I) If $0<\beta<5 \delta(0<\delta<1)$, then for all $0<\lambda<\infty$ there holds

$$
E_{\beta}(\zeta) \geqslant c(\lambda, \delta)>0 \text {. }
$$

(II) For arbitrary $0<\beta<\infty$ there exists $\lambda_{\beta}>0$ such that if $\lambda>\lambda_{\beta}$ then $E_{\beta}(\zeta) \geqslant c^{\prime}(\beta)>0$.

The constant $c(\lambda, \delta)$ of part (I) degenerates as $\delta \rightarrow 1$ and as $\lambda \rightarrow 0$; we note that (4.13) is uniformly valid as $\beta \rightarrow 0$. Part (II) is added here for completeness-we shall need only part (I) in the sequel.

PRoof. (I) Let $\zeta_{1}(x)=\lambda I_{\{|x|<a\}}$ with $a$ determined so that $\frac{1}{2} \int_{R^{3}} r^{2} \zeta_{1}(x) d x=1$. Consider the scaled functions

$$
\zeta_{\sigma}(x)=\sigma^{5} \zeta_{1}(\sigma x) \quad(0<\sigma<1) .
$$

Then

$$
\int_{R^{3}} \zeta_{\sigma} d x=\sigma^{2} \int_{R^{3}} \zeta_{1} d x, \quad \frac{1}{2} \int_{R^{3}} r^{2} \zeta_{\sigma} d x=\frac{1}{2} \int_{R^{3}} r^{2} \zeta_{1} d x
$$

and hence for sufficiently small $\sigma$ (depending only on $\lambda$ ) we have $\zeta_{\sigma} \in \mathbb{Q}_{\infty, \beta}$. An easy calculation yields

$$
\begin{aligned}
E_{\beta}\left(\zeta_{\sigma}\right) & =\sigma^{3} E\left(\zeta_{1}\right)-\sigma^{2+5 / \beta} \beta \lambda \int_{R^{3}}\left(\frac{\zeta_{1}}{\lambda}\right)^{1+1 / \beta} d x \\
& =\sigma^{3} E\left(\zeta_{1}\right)-\sigma^{2+5 / \beta} \beta \int_{R^{3}} \zeta_{1} d x .
\end{aligned}
$$

Thus, $E_{\beta}\left(\zeta_{\sigma}\right) \geqslant c(\lambda, \delta)$ for $0<\beta<5 \delta$ provided $\sigma$ is fixed small enough. Since $E_{\beta}(\zeta)>E_{\beta}\left(\zeta_{\sigma}\right)$, result (I) follows.

(II) Fix $\zeta_{0} \in \mathbb{Q}_{\infty, \beta}$. Then

$$
E_{\beta}\left(\zeta_{0}\right)=E\left(\zeta_{0}\right)-\beta \lambda^{-1 / \beta} \int_{R^{3}} \zeta_{0}^{1+1 / \beta} d x>c(\beta)>0
$$

if $\lambda \geqslant \lambda_{\beta}$ (taken large enough).

We now compute the variational conditions for the solution $\zeta$.

LEMMA 4.3. The solution asserted by Lemma 4.1 satisfies (2.17) and (2.18) for some constants $W \geqslant 0, \gamma \geqslant 0$ (uniquely determined by $\zeta$ ).

Proof. The positivity of $E_{\beta}(\zeta)$ given by Lemma 4.2 implies that meas supp $\zeta>$ 0 ; therefore, we can find $\delta_{0}>0$ such that meas $\left\{\zeta>\delta_{0}\right\}>0$. We choose bounded, measurable functions $h_{1}, h_{2}$ such that

$$
\begin{aligned}
& \operatorname{supp} h_{1}, \text { supp } h_{2} \subseteq\left\{\zeta \geqslant \delta_{0}\right\}, \\
& \int_{H} h_{1} d x=1, \quad \frac{1}{2} \int_{H} r^{2} h_{1} d x=0, \quad \int_{H} h_{2} d x=0, \quad \frac{1}{2} \int_{H} r^{2} h_{2} d x=1 ;
\end{aligned}
$$

of course, $h_{1}, h_{2}$ can be assumed to be of the form (2.1). Let $h$ be an arbitrary bounded, measurable function (of the form (2.1)) subject to the restriction that $h \geqslant 0$ a.e. on $\{\zeta<\delta\}$ for some $\delta>0$. Then $\zeta+\varepsilon \eta \in \mathbb{Q}_{\infty, \beta}^{\prime}$ for

$$
\eta=h-\left(\int_{H} h d x\right) h_{1}-\left(\frac{1}{2} \int_{H} r^{2} h d x\right) h_{2} \text {, }
$$


provided $\varepsilon>0$ is sufficiently small. Thus, by the maximality of $\zeta$ over $\mathbb{Q}_{\infty, \beta}^{\prime}$ we have

$$
\begin{aligned}
0 & \geqslant\left.(d / d \varepsilon) E_{\beta}(\zeta+\varepsilon \eta)\right|_{\varepsilon=0}=E_{\beta}^{\prime}(\zeta) \eta \\
& =E_{\beta}^{\prime}(\zeta) h-\left(\int_{H} h d x\right) E_{\beta}^{\prime}(\zeta) h_{1}-\left(\frac{1}{2} \int_{H} r^{2} h d x\right) E_{\beta}^{\prime}(\zeta) h_{2}
\end{aligned}
$$

with the Fréchet differential

$$
E_{\beta}^{\prime}(\zeta) h=\int_{H} \psi h d x-(1+\beta) \int_{H}\left(\frac{\zeta}{\lambda}\right)^{1 / \beta} h d x
$$

Now by the arbitrariness of $h$ (and $\delta$ ) we obtain the variational conditions

$$
\begin{aligned}
(1+\beta)(\zeta / \lambda)^{1 / \beta} & =\psi-\gamma-\frac{1}{2} W r^{2} \quad \text { if } \zeta>0, \\
0 & \geqslant \psi-\gamma-\frac{1}{2} W r^{2} \text { if } \zeta=0,
\end{aligned}
$$

with the Lagrange multipliers

$$
\gamma=E_{\beta}^{\prime}(\zeta) h_{1}, \quad W=E_{\beta}^{\prime}(\zeta) h_{2} .
$$

These conditions are equivalent to (2.17) and (2.18).

Finally we show that $\gamma, W \geqslant 0$. Clearly there is a sequence of points $\left(r_{n}, z_{n}\right) \in H$ such that $r_{n} \rightarrow 0, z_{n} \rightarrow \infty$ and $\zeta\left(r_{n}, z_{n}\right) \rightarrow 0$. Then the variational conditions imply that

$$
\limsup _{n \rightarrow \infty}\left\{\psi\left(r_{n}, z_{n}\right)-\gamma-\frac{1}{2} W r_{n}^{2}\right\} \leqslant 0 .
$$

Since $\psi \geqslant 0$ everywhere we conclude that $\gamma \geqslant 0$. Now take a sequence such that $r_{n} \rightarrow \infty, z_{n} \rightarrow 0$ and $\zeta\left(r_{n}, z_{n}\right) \rightarrow 0$. Then since $\psi\left(r_{n}, z_{n}\right) \rightarrow 0$ we conclude, analogously, that $W \geqslant 0$.

To prove uniqueness of the Lagrange multipliers $\gamma, W$ suppose $\gamma^{*}, W^{*}$ is another such pair, i.e., (2.17) and (2.18) hold for $\gamma^{*}, W^{*}$. This is equivalent to

$$
E_{\beta}^{\prime}(\zeta) h-\gamma^{*}\left(\int_{H} h d x\right)-W^{*}\left(\frac{1}{2} \int_{H} r^{2} h d x\right)<0
$$

for any $h$ subject to the restriction $h \geqslant 0$ a.e. on $\{\zeta<\delta\}$ for some $\delta>0$. In particular, we can take $h= \pm h_{1}$, $\pm h_{2}$ (recalling that supp $h_{i} \subset\left\{\zeta>\delta_{0}\right\}$ ) and conclude that $\gamma^{*}=E^{\prime} h_{1}, W^{*}=E^{\prime} h_{2}$.

LEMMA 4.4. For the solution $\zeta$ there holds

$$
E(\zeta)=\frac{1}{2} \int_{H} \frac{1}{r^{2}}\left(\psi_{r}^{2}+\psi_{z}^{2}\right) d x<\infty
$$

This lemma is necessary because $\zeta$ is not yet known to have compact support.

PROOF. Recall that $E(\zeta)=-\int_{H} r^{-2} \psi L \psi d x$. Integrating by parts on the domain

$$
D_{a}=\{(r, z) \in H ; 0 \leqslant r<a,|z|<a\}
$$

we obtain

$$
-\iint_{D_{a}} \frac{1}{r} \psi L \psi d r d z=\iint_{D_{a}} \frac{1}{r}\left(\psi_{r}^{2}+\psi_{z}^{2}\right) d r d z-\int_{\partial D_{a}} \psi \frac{1}{r} \frac{\partial \psi}{\partial \nu} d s
$$


( $\nu$ is the outer unit normal on $\partial D_{n}$, and $d s$ is arc length). Therefore, to prove (4.14) it suffices to show that the boundary integral tends to zero as $a \rightarrow \infty$.

We claim that for some $\varepsilon_{0}>0$,

$$
\int_{\partial D_{a}} \psi \frac{1}{r}|\nabla \psi| d s=O\left(a^{-e_{0}}\right) \quad \text { as } a \rightarrow \infty
$$

We know by Lemma 3.4 that

$$
\psi(x) \leqslant C(1+r)^{-1+e} \quad(0<\varepsilon<1) .
$$

Also, by the calculations of Lemma 1.1 (specifically, combining the curl of (1.23) with (1.26)), we have

$$
\frac{1}{r}|\nabla \psi(x)|<\frac{1}{4 \pi} \int_{R^{3}} \frac{r^{\prime} \zeta\left(x^{\prime}\right) d x^{\prime}}{\left|x-x^{\prime}\right|^{2}} .
$$

We write, for $0<\delta<1$,

$$
V_{1}(x)=\frac{1}{4 \pi} \int_{\left|x-x^{\prime}\right|<a^{8}} \frac{r^{\prime} \zeta\left(x^{\prime}\right) d x^{\prime}}{\left|x-x^{\prime}\right|^{2}}, \quad V_{2}(x)=\frac{1}{4 \pi} \int_{\left|x-x^{\prime}\right|>a^{8}} \frac{r^{\prime} \zeta\left(x^{\prime}\right) d x^{\prime}}{\left|x-x^{\prime}\right|^{2}}
$$

we estimate these separately.

Using the fact that

$$
\int_{R^{3}} r^{\prime} \zeta\left(x^{\prime}\right) d x^{\prime} \leqslant\left\{\int_{R^{3}}\left(x^{\prime}\right) d x^{\prime}\right\}^{1 / 2}\left\{\int_{R^{3}} r^{\prime 2} \zeta\left(x^{\prime}\right) d x^{\prime}\right\}^{1 / 2}<\sqrt{2},
$$

it is clear that $V_{2}(x)<C a^{-2 \delta}$. Thus,

$$
\begin{aligned}
& \int_{\substack{r=a \\
|z|<a}} \psi V_{2} d z<C(1+a)^{-1+e} a^{-2 \delta} \int_{-a}^{a} d z<C a^{-2 \delta+e}, \\
& \int_{\substack{z= \pm a \\
0<r<a}} \psi V_{2} d r<C a^{-2 \delta} \int_{0}^{a}(1+r)^{-1+e} d r \leqslant C a^{-2 \delta+e} .
\end{aligned}
$$

Hence, fixing $\varepsilon<2 \delta$, we conclude

$$
\int_{\partial D_{a}} \psi V_{2} d s=O\left(a^{-\varepsilon_{0}}\right)
$$

We now estimate the corresponding expressions in $V_{1}$. It follows from the axisymmetry of $\zeta(x)$ combined with the fact that $\zeta(r, z)$ is a nonincreasing function of $z$ for $z>0$ that

$$
\int_{\left|x-x^{\prime}\right|<a^{\delta}} r^{\prime} \zeta\left(x^{\prime}\right) d x^{\prime}<C_{0}\left(1+\frac{r}{a^{\delta}}\right)^{-1}\left(1+\frac{|z|}{a^{\delta}}\right)^{-1}
$$

for all $(r, z) \in H$. Indeed, since for $r>2 a^{\delta}$,

$$
\begin{aligned}
\operatorname{meas}\left\{x^{\prime} \in R^{3} ;\right. & \left.\left|x-x^{\prime}\right|<a^{\delta}\right\} \\
& <\left(C a^{\delta} / r\right) \operatorname{meas}\left\{x^{\prime} \in R^{3} ;\left|r-r^{\prime}\right|<a^{\delta},\left|z-z^{\prime}\right|<a^{\delta}\right\},
\end{aligned}
$$


we find (using $\left.\zeta\left(x^{\prime}\right)=\zeta\left(r^{\prime}, z^{\prime}\right)\right)$ that

$$
\int_{\left|x-x^{\prime}\right|<a^{\delta}} r^{\prime} \zeta\left(x^{\prime}\right) d x^{\prime} \leqslant \frac{C a^{\delta}}{r} \int_{\substack{\left|r-r^{\prime}\right|<a^{\delta} \\\left|z-z^{\prime}\right|<a^{\delta}}} r^{\prime} \zeta\left(x^{\prime}\right) d x^{\prime} ;
$$

in turn, for $z>2 a^{\delta}$ (using $\zeta\left(r^{\prime}, z^{\prime}\right) \downarrow$ as $z^{\prime} \uparrow, z^{\prime}>0$ )

$$
\int_{\substack{\left|r-r^{\prime}\right|<a^{\delta} \\\left|z-z^{\prime}\right|<a^{\delta}}} r^{\prime} \zeta\left(x^{\prime}\right) d x^{\prime} \leqslant \frac{C a^{\delta}}{z} \int_{\left|r-r^{\prime}\right|<a^{\delta}} r^{\prime} \zeta\left(x^{\prime}\right) d x^{\prime} .
$$

Thus, (4.18) follows.

Also, since $\zeta=C\left(u^{+}\right)^{\beta} \leqslant C \psi^{\beta}$, we have

$$
\sup _{\left|x-x^{\prime}\right|<a^{\delta}} r^{\prime} \zeta\left(x^{\prime}\right) \leqslant C_{1} a^{1-\mu} \quad \text { if } x \in \partial D_{a}
$$

where $0<\mu<1$ (depends on $\beta$ ). For $r=a,|z|<a$, consider the problem to maximize $V_{1}(x)$ as a functional of $r^{\prime} \zeta\left(x^{\prime}\right)$ subject to (4.18) and (4.19). Clearly, the maximum occurs when $r^{\prime} \zeta\left(x^{\prime}\right)=C_{1} a^{1-\mu} I_{\left\{\left|x-x^{\prime}\right|<\rho\right\}}$ where $\rho$ is determined so that equality holds in (4.18). Computing $V_{1}(x)$ in this case we obtain

$$
V_{1}(x) \leqslant C a^{1 / 3-2 \mu / 3+\delta / 3}\left(1+|z| / a^{\delta}\right)^{-1 / 3} .
$$

Thus,

$$
\begin{aligned}
\int_{\substack{r=a \\
|z|<a}} \psi V_{1} d z & \leqslant C(1+a)^{-1+\varepsilon} a^{1 / 3-2 \mu / 3+\delta / 3} \int_{-a}^{a}\left(1+\frac{|z|}{a^{\delta}}\right)^{-1 / 3} d z \\
& \leqslant C a^{-2 \mu / 3+2 \delta / 3+e} .
\end{aligned}
$$

Similarly, for $z= \pm a, 0<r \leqslant a$, we find

$$
V_{1}(x) \leqslant C a^{1 / 3-2 \mu / 3+\delta / 3}\left(1+r / a^{\delta}\right)^{-1 / 3},
$$

and thus

$$
\int_{\substack{z= \pm a \\ 0<r<a}} \psi V_{1} d r \leqslant C a^{-2 \mu / 3+5 \delta / 3+(1-\delta) e}
$$

Fixing $\varepsilon$ and $\delta$ sufficiently small we find

$$
\int_{\partial D_{a}} \psi V_{1} d s=O\left(a^{-e_{0}}\right)
$$

This proves the claim and, hence, the lemma.

LEMMA 4.5. In the notation of Lemma 4.2(I), there holds

$$
W \geqslant \frac{1}{2} c(\lambda, \delta)>0
$$

as a consequence, $\zeta \in \mathbb{Q}_{\infty, \beta}$.

In the sequel we shall assume that $0<\beta<5 \delta(0<\delta<1)$ and no longer specify the dependence of any constants on $\delta$ (fixed). 
Proof. By Lemma 4.4 (which assures the hypothesis of Lemma 3.1) and the remark following Lemma 3.2, we may apply the identity (3.10) to the solution $\zeta$ and obtain

$$
W \geqslant \frac{1}{2} E(\zeta)
$$

since $J(u)>0$. The assertion (4.20) now follows immediately from (4.13), using $E_{\beta}(\zeta)<E(\zeta)$

Since $W$, the Lagrange multiplier for the constraint (4.1), must equal zero if strict inequality holds in (4.1) we now conclude that the equality (2.2) always holds on a solution; that is, $\zeta \in \mathbb{Q}_{\infty, \beta}$.

To complete the proof of Theorem 2.2 it remains to show that the solution obtained has compact support.

LEMMA 4.6. If $(r, z) \in \operatorname{supp} \zeta$ then

$$
\frac{1}{2} r^{2}|z|<E(\zeta) / \pi W^{2}
$$

Proof. Suppose $z>0$; then for all $0<z^{\prime}<z$, since $\left(r, z^{\prime}\right) \in \operatorname{supp} \zeta$, we have

$$
\psi\left(r, z^{\prime}\right) \geqslant \frac{1}{2} W r^{2}+\gamma \geqslant \frac{1}{2} W r^{2} .
$$

Then, since $\psi\left(0, z^{\prime}\right)=0$,

$$
\frac{W r^{2}}{2}<\int_{0}^{r} \psi_{r^{\prime}}\left(r^{\prime}, z^{\prime}\right) d r^{\prime}
$$

Integrating in $z^{\prime}$ we get

$$
\begin{aligned}
\frac{W r^{2} z}{2} & <\int_{0}^{r} \int_{0}^{z} \psi_{r^{\prime}}\left(r^{\prime}, z^{\prime}\right) d r^{\prime} d z^{\prime} \\
& <\left\{\int_{0}^{r} \int_{0}^{z} r^{\prime} d r^{\prime} d z^{\prime}\right\}^{1 / 2}\left\{\int_{0}^{r} \int_{0}^{z} \frac{1}{r^{\prime}} \psi_{r^{2}}^{2}\left(r^{\prime}, z^{\prime}\right) d r^{\prime} d z^{\prime}\right\}^{1 / 2} \\
& \leqslant\left(r^{2} z / 2\right)^{1 / 2}(E(\zeta) / \pi)^{1 / 2}
\end{aligned}
$$

so (4.22) follows.

LEMMA 4.7. There is $r^{*}(\lambda)<\infty$ such that

$$
r<r^{*}(\lambda) \text { for all }(r, z) \in \operatorname{supp} \zeta \text {. }
$$

Proof. By (4.20) we have

$$
\psi(r, z)>\frac{1}{2} W r^{2}+\gamma>c_{\lambda} r^{2} ; \quad c_{\lambda}>0 .
$$

Combining this with the estimate (3.19) (recall that $N(\zeta)<C_{\lambda}$ ) we find

$$
c_{\lambda} r^{2}<\psi(r, z)<C_{\lambda, e^{-1+e}} \quad(0<\varepsilon<1) .
$$

Now fixing $\varepsilon$ we have $r^{3-\varepsilon}<\tilde{C}_{\lambda}$, as required.

In order to prove the analogous result in $z$ we require a further lemma which is stated in Fraenkel and Berger [13, p. 39]; for convenience we shall give the proof. 
LEMMA 4.8. For $0<r<\rho$ there holds

$$
\underset{\substack{0<r^{\prime}<\rho \\-\infty<z^{\prime}<\infty}}{\int} G\left(r, z, r^{\prime}, z^{\prime}\right) r^{\prime} d r^{\prime} d z^{\prime} \leqslant C r^{2} \rho^{2}
$$

$C$ is an absolute positive constant.

Proof. Writing $s=\left[\left(r-r^{\prime}\right)^{2}+\left(z-z^{\prime}\right)^{2}\right]^{1 / 2}$ we consider separately the contributions for $s \leqslant r / 2$ and $s>r / 2$ as in the estimates of §3. According to Lemma 3.3,

$$
\begin{gathered}
\iint_{s<r / 2} G\left(r, z, r^{\prime}, z^{\prime}\right) r^{\prime} d r^{\prime} d z^{\prime} \leqslant C r^{2} \iint_{s<r / 2} \log \frac{r}{s} d r^{\prime} d z^{\prime}=C r^{4} \leqslant C r^{2} \rho^{2} . \\
\qquad \int_{\substack{s>r / 2 \\
0<r^{\prime}<\rho}} G\left(r, z, r^{\prime}, z^{\prime}\right) r^{\prime} d r^{\prime} d z^{\prime} \leqslant C r^{2} \int_{\substack{s>r / 2 \\
0<r^{\prime}<\rho}} \frac{r^{\prime 3}}{s^{3}} d r^{\prime} d z^{\prime} .
\end{gathered}
$$

It is easily verified that for $s>r / 2$ there holds

$$
r^{3} / s^{3} \leqslant C r^{3} /\left[\left(z-z^{\prime}\right)^{2}+\left(r+r^{\prime}\right)^{2}\right]^{3 / 2}
$$

Hence, it now suffices to estimate

$$
\iint_{0<r^{\prime}<\rho} \frac{r^{3}}{\left[\left(z-z^{\prime}\right)^{2}+\left(r+r^{\prime}\right)^{2}\right]^{3 / 2}} d r^{\prime} d z^{\prime}=2 \int_{0}^{\rho} \frac{r^{3}}{\left(r+r^{\prime}\right)^{2}} d r^{\prime} \leqslant C \rho^{2} .
$$

LEMMA 4.9. There is $z^{*}(\lambda)<\infty$ such that

$$
|z| \leqslant z^{*}(\lambda) \text { for all }(r, z) \in \operatorname{supp} \zeta .
$$

Proof. By Lemma 4.6 we have $r^{2} z \leqslant C_{\lambda}$ (we take $z>0$ ); thus, to prove (4.26) we may assume that $r<z$, say. Let $\rho$ be defined by $\rho^{2} z / 2=C_{\lambda}$. Then

$$
\begin{aligned}
\psi(r, z)= & \iint_{H} G\left(r, z, r^{\prime}, z^{\prime}\right) \zeta\left(r^{\prime}, z^{\prime}\right) r^{\prime} d r^{\prime} d z^{\prime} \\
\leqslant & \sup _{0<r^{\prime}<\rho} \zeta\left(r^{\prime}, z^{\prime}\right) \int_{0<r^{\prime}<\rho} G\left(r, z, r^{\prime}, z^{\prime}\right) r^{\prime} d r^{\prime} d z^{\prime} \\
& +\int_{r^{\prime}>\rho} G\left(r, z, r^{\prime}, z^{\prime}\right) \zeta\left(r^{\prime}, z^{\prime}\right) r^{\prime} d r^{\prime} d z^{\prime} .
\end{aligned}
$$

Now (2.17), (2.18) and the fact that $\sup _{H} \psi \leqslant C_{\lambda}$ imply $\sup _{H} \zeta \leqslant C_{\lambda}$. If $r^{\prime}>\rho$ then, since

$$
r^{\prime 2}\left|z^{\prime}\right| \leqslant C_{\lambda}=\rho^{2} z / 2 \leqslant r^{\prime 2} z / 2,
$$

we find that $\left|z^{\prime}\right| \leqslant z / 2$ whenever $\left(r^{\prime}, z^{\prime}\right) \in \operatorname{supp} \zeta$. Thus, in the notation of Lemma 3.3, there holds $s>z / 2>r / 2$ for all $\left(r^{\prime}, z^{\prime}\right) \in \operatorname{supp} \zeta$ with $r^{\prime}>\rho$. Applying estimate (3.12) in this case yields

$$
\begin{aligned}
& \int_{r^{\prime}>\rho} G\left(r, z, r^{\prime}, z^{\prime}\right) \zeta\left(r^{\prime}, z^{\prime}\right) r^{\prime} d r^{\prime} d z^{\prime} \\
& \quad \leqslant C r^{2} \int_{r^{\prime}>\rho} \frac{r^{\prime 2}}{s^{3}} \zeta\left(r^{\prime}, z^{\prime}\right) r^{\prime} d r^{\prime} d z^{\prime}<\frac{C r^{2}}{z^{3}} \iint_{H} r^{\prime 2} \zeta\left(r^{\prime}, z^{\prime}\right) r^{\prime} d r^{\prime} d z^{\prime} .
\end{aligned}
$$


Returning to the estimation of $\psi(r, z)$ we now have, using Lemma 4.8,

$$
\psi(r, z) \leqslant C_{\lambda} r^{2} \rho^{2}+C r^{2} / z^{3} \leqslant C_{\lambda} r^{2} / z+C r^{2} / z^{3} .
$$

The required result follows upon combining (4.24) and (4.27).

REMARK. The method of the above proof is adopted from [13].

5. Proof of Theorem 2.1. We shall give the proof in the form of two lemmas.

LEMMA 5.1. There exists $\zeta \in \mathbb{Q}_{\lambda}$ such that (2.6) holds, $\zeta$ has compact support, and $\zeta(r, z)$ is a nonincreasing function of $z$ for $z>0$.

PRoof. Let $\zeta_{\beta}$ denote the solution obtained in Theorem 2.2 for the penalized problem with a prescribed $\lambda$ (and $0<\beta<1$, say). We know from Lemmas 4.7 and 4.9 that

$$
\operatorname{supp} \zeta_{\beta} \subseteq D \equiv\left\{(r, z) \in H ; 0 \leqslant r \leqslant r^{*}(\lambda),|z|<z^{*}(\lambda)\right\}
$$

independent of $\beta$.

Applying (4.6) to $\zeta_{\beta}$ and recalling that $E_{\beta}\left(\zeta_{\beta}\right)>0$ we find that

$$
\beta \int_{H}\left(\frac{\zeta_{\beta}}{\lambda}\right)^{1+1 / \beta} d x \leqslant C_{\lambda} .
$$

Using the notation introduced in (3.18) this becomes

$$
N\left(\zeta_{\beta}\right) / \lambda \leqslant\left(C_{\lambda} / \beta\right)^{\beta /(1+\beta)},
$$

which in turn implies that

$$
N\left(\zeta_{\beta}\right) \leqslant \lambda+o(1) \text { as } \beta \rightarrow 0 .
$$

Now if $\beta<\alpha$, for any fixed $0<\alpha<1$, then we can estimate

$$
\begin{aligned}
\left\|\zeta_{\beta}\right\|_{L^{1+1 / \alpha}} & \leqslant\left\|\zeta_{\beta}\right\|_{L^{1}}^{1-a}\left\|\zeta_{\beta}\right\|_{L^{1+1 / \beta}}^{a} \quad(a=(1+\beta) /(1+\alpha)) \\
& \leqslant[\lambda+o(1)]^{a} \text { as } \beta \rightarrow 0 .
\end{aligned}
$$

Therefore, there exists a sequence $\beta_{j} \rightarrow 0$ (constructed by the usual diagonal process) such that

$$
\zeta_{\beta_{j}} \rightarrow \zeta \text { weakly in } L^{1+1 / \alpha}(D) \text { for every } 0<\alpha<1 \text {. }
$$

Furthermore, by (5.4),

$$
\|\zeta\|_{L^{1+1 / \alpha}} \leqslant \liminf _{j \rightarrow \infty}\left\|\zeta_{\beta_{j}}\right\|_{L^{1+1 / \alpha}} \leqslant \lambda^{1 /(1+\alpha)}
$$

now taking $\alpha \rightarrow 0$ we conclude

$$
\text { ess sup } \zeta=\lim _{\alpha \rightarrow 0}\|\zeta\|_{L^{1+1 / \alpha}} \leqslant \lambda .
$$

Also, by virtue of (5.1), it follows easily that

$$
\int_{H} \zeta d x \leqslant 1, \quad \frac{1}{2} \int_{H} r^{2} \zeta d x=1 .
$$

Thus, $\zeta \in \mathbb{Q}_{\lambda}$, supp $\zeta \subseteq D$, and $\zeta(r, z)$ is a nonincreasing function of $z$ for $z>0$ (a property inherited from the $\zeta_{\beta}$ solutions). 
Finally, we must show that (2.6) holds. In light of (5.1) it is immediate from standard arguments that

$$
\lim _{j \rightarrow \infty} E\left(\zeta_{\beta_{j}}\right)=E(\zeta)
$$

For any $\tilde{\zeta} \in Q_{\lambda}$ we have

$$
E(\zeta)=\lim _{j \rightarrow \infty} E\left(\zeta_{\beta_{j}}\right) \geqslant \lim _{j \rightarrow \infty} E_{\beta_{j}}\left(\zeta_{\beta_{j}}\right) \geqslant \lim _{j \rightarrow \infty} E_{\beta_{j}}(\tilde{\zeta})=E(\tilde{\zeta}) ;
$$

the last equality follows since

$$
\lim _{\beta \rightarrow 0} \beta \lambda \int_{H}\left(\frac{\tilde{\zeta}}{\lambda}\right)^{1+1 / \beta} d x=0 .
$$

This establishes (2.6) and completes the proof.

We now take the "limit" as $\beta \rightarrow 0$ of the variational conditions for $\zeta_{\beta}$ to obtain the analogous conditions for $\zeta$ :

LEMMA 5.2. There exist constants $W>0, \gamma>0$ such that (2.7) and (2.8) hold.

Proof. Let $W_{j}, \gamma_{j}, \psi_{j}, u_{j}$ denote the quantities in the statement of Theorem 2.2 associated with $\zeta_{\beta_{j}}$. Since $W_{j}, \gamma_{j} \geqslant 0$, and as a consequence of (3.10), $W_{j}+\gamma_{j} \leqslant$ $2 E\left(\zeta_{\beta_{j}}\right) \leqslant C_{\lambda}$, we may assume (by taking a subsequence) that $W_{j} \rightarrow W$ and $\gamma_{j} \rightarrow \gamma$. Then, $\gamma>0$ and, by (4.20), $W \geqslant c_{\lambda}>0$.

In view of (5.1) and (5.5),

$$
\psi_{j}(x)=\int_{R^{3}} K\left(x, x^{\prime}\right) \zeta_{\beta_{j}}\left(x^{\prime}\right) d x^{\prime}
$$

converges pointwise on $R^{3}$ to

$$
\psi(x)=\int_{R^{3}} K\left(x, x^{\prime}\right) \zeta\left(x^{\prime}\right) d x^{\prime}
$$

Hence, $u_{j}(x) \rightarrow u(x)$ pointwise on $R^{3}$. Also, according to the variational condition

$$
\zeta_{\beta_{j}}(x)=\lambda\left\{u_{j}^{+}(x) /\left(1+\beta_{j}\right)\right\}^{\beta_{j}},
$$

we have

$$
\lim _{j \rightarrow \infty} \zeta_{\beta_{j}}(x)= \begin{cases}\lambda & \text { if } u(x)>0, \\ 0 & \text { if } u(x)<0 .\end{cases}
$$

Furthermore, since $u_{z}=\psi_{z}<0$ for $z>0$ (note that $u(r, z) \in C^{1}(H)$ ) we see that meas $\left\{x \in R^{3} ; u(x)=0\right\}=0$. Thus, the function $\lambda I_{\{u(x)>0\}}$ is a pointwise limit of $\zeta_{\beta_{j}}(x)$ for a.a. $x \in R^{3}$; hence (by a straightforward application of the bounded convergence theorem), the function $\lambda I_{\{u(x)>0\}}$ coincides with the weak limit $\zeta(x)$. This completes the proof.

REMARK 1. We shall later need the fact that

$$
\text { if } \gamma>0 \text { then } \int_{R^{3}} \zeta(x) d x=1
$$

To prove it suppose $\int_{R^{3}} \zeta(x) d x<1$. Then also

$$
\int_{R^{3}} \zeta_{\beta_{j}}(x) d x<1 \text { for } j \text { sufficiently large. }
$$


But then we can take in the derivation of the variational condition, as given in the proof of Lemma 4.3,

$$
\eta=h-\left(\frac{1}{2} \int_{H} r^{2} h d x\right) h_{2}
$$

this leads to the (unique) choice of $\gamma_{j}=0$. Hence also $\gamma=\lim \gamma_{j}=0$.

REMARK 2. We shall now show that

$$
\int \zeta d x<1 \text { if } \lambda \text { is sufficiently small; }
$$

consequently $\gamma=0$ if $\lambda$ is small enough. Let $\zeta_{0}(r, z)=\lambda_{\Omega_{0}}$ where $\Omega_{0}$ is a disc with center $(2 R, 0)$ and radius $R, R=c / \lambda^{1 / 5}$. Then for a suitable choice of $c$ (independent of $\lambda$ ), $\zeta_{0} \in \mathbb{Q}_{\lambda}$ and one can compute (cf. [14]) that

$$
E\left(\zeta_{0}\right)>C \lambda^{3 / 5} \quad(C>0) .
$$

Next, by Lemma 4.6 and the inequality $W>\frac{1}{2} E(\zeta)$,

$$
r^{2}|z|<c / E(\zeta)<c / \lambda^{3 / 5} \quad \text { (where (5.10) is used) }
$$

provided $(r, z) \in \operatorname{supp} \zeta$. Thus

$$
|z| \leqslant\left(C / \lambda^{3 / 5}\right)\left(1 / r^{2}\right) \quad \text { if }(r, z) \in \operatorname{supp} \zeta .
$$

If we utilize the inequality $\zeta<\lambda$ in the proof of Lemma 4.9, we obtain the inequality (4.27)

$$
\psi(r, z) \leqslant\left(c \lambda^{2 / 5} /|z|+C /|z|^{3}\right) r^{2} .
$$

Since, on the other hand,

$$
\psi(r, z)>W r^{2} / 2 \text { if }(r, z) \in \operatorname{supp} \zeta,
$$

we get

$$
\lambda^{2 / 5} /|z|+1 /|z|^{3}>c W>c \lambda^{3 / 5} \quad \text { if }(r, z) \in \operatorname{supp} \zeta
$$

that is, setting

$$
Z_{\lambda}=2+\sup \{|z| ;(r, z) \in \operatorname{supp} \zeta\}
$$

there holds

$$
Z_{\lambda}<C / \lambda^{1 / 5}
$$

We now compute, using (5.11),

$$
\int_{r<\sqrt{2}} \zeta d x=2 \pi \lambda \int_{\substack{r<\sqrt{2} \\ \zeta>0}} r d r d z \leqslant c \lambda \int_{\varepsilon}^{\sqrt{2}} \frac{r d r}{\lambda^{3 / 5} r^{2}}=c \lambda^{2 / 5} \log \frac{1}{\varepsilon}
$$

where $\varepsilon^{2} \lambda^{3 / 5}=C / Z_{\lambda}$. Hence

$$
\int_{r<\sqrt{2}} \zeta d x<c \lambda^{2 / 5} \log \frac{1}{\lambda}
$$

Suppose now that (5.9) is not true, that is, $\int \zeta d x=1$. Since also $\frac{1}{2} \int r^{2} \zeta d x=1$, we must have $\int_{r>\sqrt{2}} \zeta d x<\int_{r<\sqrt{2}} \zeta d x$ so that, by (5.13), $\int \zeta d x<1$, a contradiction. 
6. Asymptotic results; preliminary estimates. The rest of the paper is devoted to obtaining asymptotic estimates on the solution when $\lambda \rightarrow \infty$. We shall denote the solution constructed in Theorem 1.1 by $\zeta=\zeta_{\lambda}$ and set

$$
B=B_{\lambda}=\left\{(r, z) ; \zeta_{\lambda}(r, z)=\lambda\right\} \text {. }
$$

The constraint (2.2) gives

$$
\left|B_{\lambda}\right| \leqslant 1 / 2 \pi \lambda
$$

where $|A|$ denotes the measure of a set $A$.

From a general theorem of Caffarelli and Friedman [9] it follows that $B_{\lambda}$ consists of a finite number of components and the boundary $z= \pm Z(r)$ of each component is analytic. As asserted by Benjamin [4],

$$
B_{\lambda} \text { is connected. }
$$

In fact, otherwise we can write $B_{\lambda}=A_{1} \cup A_{2}$ where each $A_{i}$ consists of a finite number of components, $\overline{A_{1}} \cap \overline{A_{2}}=\varnothing$, and $A_{1}$ lies to the left of $A_{2}$. By moving $A_{1}$ and $A_{2}$ toward each other (preserving the constraints (2.2), (2.3)) one obtains a function $\tilde{\zeta}$ such that $E(\tilde{\zeta})>E\left(\zeta_{\lambda}\right)$, a contradiction.

LEMMA 6.1. For all $\lambda$ sufficiently large,

$$
E=E\left(\zeta_{\lambda}\right) \geqslant(\log \lambda) /\left(8 \sqrt{2} \pi^{2}\right)-C,
$$

where $C$ is a constant independent of $\lambda$.

Proof. We shall use formulas (3.15)-(3.17). Let

$$
\zeta_{0}(r, z)=\lambda I_{D_{e}(\sqrt{2}, 0)}
$$

where $D_{e}(\sqrt{2}, 0)$ is the $\operatorname{disc}(r-\sqrt{2})^{2}+z^{2}<\varepsilon^{2}$. Then

$$
\int \zeta_{0}(x) d x=1 \text { if } 2 \pi \sqrt{2} \varepsilon^{2} \lambda=1
$$

i.e., if

$$
\varepsilon^{2} \lambda=1 /\left(2 \sqrt{2} \pi^{2}\right) .
$$

We shall compute $E\left(\zeta_{0}\right)$. For this purpose take first, in $G\left(r, z, r^{\prime}, z^{\prime}\right),\left(r^{\prime}, z^{\prime}\right)=$ $(\sqrt{2}, 0)$ and introduce new coordinates about $(\sqrt{2}, 0): r=\sqrt{2}+\varepsilon s, z=\varepsilon t$, $\xi=\sqrt{s^{2}+t^{2}}$. Then $0 \leqslant \xi \leqslant 1$ if $(r, z) \in \operatorname{supp} \zeta_{0}$. In terms of the new variables, we find (recall (3.15)-(3.17))

$$
\begin{aligned}
& k^{\prime 2}=\frac{\varepsilon^{2} t^{2}+\varepsilon^{2} s^{2}}{\varepsilon^{2} t^{2}+(2 \sqrt{2}+\varepsilon s)^{2}}=\frac{\varepsilon^{2} \xi^{2}}{8}\{1+O(\varepsilon)\} \\
& k^{2}=1+O(\varepsilon), \quad 4 / k^{\prime}=(8 \sqrt{2} / \varepsilon \xi)\{1+O(\varepsilon)\}
\end{aligned}
$$

Hence

$$
(2 / k-k) K(k)-2 E(k) / k=\log (8 \sqrt{2} / \varepsilon \xi)-2+O\left(\varepsilon \log \varepsilon^{-1}\right) .
$$

Using $\left(r r^{\prime}\right)^{1 / 2}=\sqrt{2}+O(\varepsilon)$, we get

$$
\begin{aligned}
G(r, z, \sqrt{2}, 0) & =(\sqrt{2} / 2 \pi)[\log (8 \sqrt{2} / \varepsilon \xi)-2]+O\left(\varepsilon \log \varepsilon^{-1}\right) \\
& =(\sqrt{2} / 2 \pi) \log (1 / \varepsilon \xi)+O(1) \quad \text { as } \varepsilon \rightarrow 0 .
\end{aligned}
$$


Let $\zeta^{0}=$ delta function on the circle $r=\sqrt{2}, z=0,-\pi<\theta<\pi$, and

$$
\psi^{0}(r, z)=\iint G\left(r, z, r^{\prime}, z^{\prime}\right) \zeta^{0}\left(r^{\prime}, z^{\prime}\right) r^{\prime} d r^{\prime} d z^{\prime}=(2 \pi)^{-1} G(r, z, \sqrt{2}, 0) .
$$

Since $G\left(r, z, r^{\prime}, z^{\prime}\right)=G(r, z, \sqrt{2}, 0)+O(1)$ on supp $\zeta_{0}$, we find that

Hence

$$
\psi_{0}(r, z) \equiv \iint G\left(r, z, r^{\prime}, z^{\prime}\right) \zeta_{0}\left(r^{\prime}, z^{\prime}\right) r^{\prime} d r^{\prime} d z^{\prime}=\psi^{0}(r, z)+O(\varepsilon) .
$$

$$
\begin{aligned}
E\left(\zeta_{0}\right) & =\pi \iint \psi^{0}(r, z) \zeta_{0}(r, z) r d r d z+O(1) \\
& =\pi \iint \frac{1}{2 \pi} \frac{\sqrt{2}}{2 \pi} \log \frac{1}{\varepsilon \xi} \zeta(r, z) r d r d z+O(1) \\
& =\frac{\sqrt{2}}{4 \pi} \iint \log \frac{1}{\varepsilon \xi} \zeta(r, z) r d r d z+O(1) .
\end{aligned}
$$

Since $r=\sqrt{2}+O(\varepsilon), d r d z=2 \pi \varepsilon^{2} \xi d \xi$, we get

$$
\begin{aligned}
E\left(\zeta_{0}\right) & =\frac{\sqrt{2}}{4 \pi} \sqrt{2} 2 \pi \varepsilon^{2} \lambda \int_{0}^{1} \xi \log \frac{1}{\varepsilon \xi} d \xi+O(1) \\
& =\varepsilon^{2} \lambda\left[\frac{1}{2} \xi^{2} \log \frac{1}{\varepsilon \xi}+\frac{1}{2}\right]_{\xi=0}^{\xi=1}+O(1) \\
& =\frac{1}{2} \varepsilon^{2} \lambda \log \frac{1}{\varepsilon}+O(1)=\frac{1}{4 \sqrt{2} \pi^{2}} \log \frac{1}{\varepsilon}+O(1)
\end{aligned}
$$

by (6.4). Since

$$
\int \zeta_{0} d x=1, \quad \frac{1}{2} \int r^{2} \zeta_{0}=1+O(\varepsilon),
$$

there is an $O(\varepsilon)$-perturbation $\zeta_{1}$ of $\zeta_{0}$ which belongs to $\mathbb{Q}_{\lambda}$. Hence

$$
E(\zeta)>E\left(\zeta_{1}\right)=E\left(\zeta_{0}\right)+O(1)
$$

and (6.3) follows.

LEMMA 6.2. There is a positive constant $C$ independent of $\lambda$ such that

$$
\psi(r, 0)<\operatorname{Cr}\left[1+\log \left(1+\lambda r^{3}\right)\right] \quad(0<r<\infty)
$$

for any $\lambda>0$, where $\psi$ is the stream function corresponding to $\zeta=\zeta_{\lambda}$.

Proof. Write $\psi(r, 0)=\psi_{1}(r, 0)+\psi_{2}(r, 0)$ where

$$
\begin{aligned}
& \psi_{1}(r, 0)=\int_{\left|x-x^{\prime}\right|<r / 2} K\left(x, x^{\prime}\right) \zeta\left(x^{\prime}\right) d x^{\prime} \quad(x=(r, 0,0)), \\
& \psi_{2}(r, 0)=\int_{\left|x-x^{\prime}\right|>r / 2} K\left(x, x^{\prime}\right) \zeta\left(x^{\prime}\right) d x^{\prime} .
\end{aligned}
$$

To estimate $\psi_{2}$ notice that

$$
K\left(x, x^{\prime}\right) \leqslant r r^{\prime} /\left|x-x^{\prime}\right| \leqslant 3 r \quad \text { if }\left|x-x^{\prime}\right|>r / 2 .
$$

Thus $\psi_{2}(r, 0) \leqslant 3 r$. 
To estimate $\psi_{1}$ notice that

$$
K\left(x, x^{\prime}\right) \leqslant C r^{2} /\left|x-x^{\prime}\right| \text { if }\left|x-x^{\prime}\right|<r / 2
$$

and hence

$$
\psi_{1}(r, 0) \leqslant C r^{2} \int_{\substack{\left|r-r^{\prime}\right|<r / 2 \\\left|z^{\prime}\right|<r / 2}} \int_{0}^{2 \pi} \frac{r^{\prime} d \theta}{\left[z^{\prime 2}+r^{2}+r^{\prime 2}-2 r r^{\prime} \cos \theta\right]^{1 / 2}} d r^{\prime} d z^{\prime} .
$$

By [14] we have

$$
\int_{0}^{2 \pi} \frac{r^{\prime} d \theta}{\left[z^{\prime 2}+r^{2}+r^{\prime 2}-2 r r^{\prime} \cos \theta\right]^{1 / 2}} \leqslant C \log \frac{C r}{\left[z^{\prime 2}+\left(r-r^{\prime}\right)^{2}\right]^{1 / 2}}
$$

and then, as in [14],

$$
\psi_{1}(r, 0) \leqslant C r^{2} r^{-1} \log \lambda r^{3} \quad \text { provided } \lambda r^{3}>c
$$

for some positive constant $c$. On the other hand, if $\lambda r^{3} \leqslant c$ then we simply use

$$
\psi_{1}(r, 0)<C r^{2} \lambda \int_{\left|x-x^{\prime}\right|<r / 2} \frac{d x^{\prime}}{\left|x-x^{\prime}\right|}<C \lambda r^{4} .
$$

The assertion (6.5) follows by putting together the estimates on $\psi_{1}, \psi_{2}$.

Define

$$
R_{0}=\inf \{r ;(r, 0) \in \operatorname{supp} \zeta\}, \quad R_{1}=\sup \{r ;(r, 0) \in \operatorname{supp} \zeta\} .
$$

Note that (2.3) implies trivially

$$
R_{0}^{2} / 2 \leqslant 1 \leqslant R_{1}^{2} / 2 \text {. }
$$

LEMMA 6.3. There holds

$$
R_{1} \leqslant C
$$

where $C$ is a constant independent of $\lambda, \lambda>1$.

Proof. Since $u=0$ on $\partial B_{\lambda}$,

$$
W R_{1}^{2} / 2+\gamma=\psi\left(R_{1}, 0\right) .
$$

Recalling that $\gamma \geqslant 0$ and using Lemma 6.2, we get

$$
W R_{1}^{2} / 2 \leqslant C R_{1} \log \left(2+\lambda R_{1}^{3}\right) \text {. }
$$

From Lemmas 3.2 and 6.1 we have

$$
2 W=E+3 J(u) \geqslant E \geqslant C \log \lambda
$$

if $\lambda$ is sufficiently large. Substituting this estimate into (6.8) we get

$$
R_{1} \log \lambda \leqslant C \log \left(\lambda R_{1}^{3}\right)
$$

if $\lambda$ is sufficiently large (say $\lambda>\lambda_{0}$ ), and (6.7) follows. The proof for $1<\lambda<\lambda_{0}$ is similar, since $E\left(\zeta_{\lambda}\right) \geqslant c>0, c$ independent of $\lambda$.

LEMMA 6.4. There holds

$$
J(u) \leqslant C
$$

where $J=J(u)$ is defined by (3.8) with $F(t)=\lambda t^{+}$and $C$ is a constant independent of $\lambda$. 
Proof. By (3.9) (with $\beta=0$ ),

$$
J(u)=2 \pi \lambda \int_{B} u r d r d z=2 \pi \int_{B} \int_{r} \frac{1}{r}\left(u_{r}^{2}+u_{z}^{2}\right) d r d z .
$$

Since $R_{1}<C$ we get

$$
Q \leqslant C J \quad \text { where } Q=\iint_{B}\left(u_{r}^{2}+u_{z}^{2}\right) d r d z .
$$

We recall the Poincaré inequality

$$
\iint_{B} u^{2} d r d z<\frac{|B|}{2 \pi} \int_{B} \int_{r}\left(u_{r}^{2}+u_{z}^{2}\right) d r d z \quad \text { if }\left.u\right|_{\partial B}=0 .
$$

We use it to derive

$$
\begin{aligned}
\frac{J}{2 \pi \lambda} & <C \int_{B} u d r d z<C|B|^{1 / 2}\left(\int_{B} \int^{2} d r d z\right)^{1 / 2} \\
& <C|B|\left(\int_{B} \int_{r}\left(u_{r}^{2}+u_{z}^{2}\right) d r d z\right)^{1 / 2} \\
& =C|B| Q^{1 / 2}<C|B| J^{1 / 2}, \text { by }(6.11)
\end{aligned}
$$

Recalling (6.1), assertion (6.10) now follows.

From Lemmas 3.2, 6.1 and 6.4 we deduce

THEOREM 6.5. As $\lambda \rightarrow \infty$,

$$
\begin{aligned}
W & =E / 2+O(1), \\
\gamma & =3 E / 2+O(1) .
\end{aligned}
$$

From (6.12) and Lemma 6.1 it follows that $\gamma>0$ if $\lambda$ is sufficiently large. Hence, by Remark 1 at the end of $\S 5$ :

COROLlaRY 6.6. If $\lambda$ is sufficiently large then

$$
\left|B_{\lambda}\right|=1 /(2 \pi \lambda) \text {. }
$$

LEMMA 6.7. If $\lambda$ is sufficiently large then

$$
R_{0} \geqslant c
$$

where $c$ is a positive constant independent of $\lambda$.

Proof. We have

$$
\gamma<\gamma+W R_{0}^{2} / 2=\psi\left(R_{0}, 0\right)<c R_{0}\left(1+\log \left(1+\lambda R_{0}^{3}\right)\right)
$$

where Lemma 6.2 was used. Since by (6.13) and (6.3),

$$
\gamma>c_{0} \log \lambda \quad\left(c_{0}>0\right)
$$

where $\lambda$ is sufficiently large and $c_{0}$ is independent of $\lambda$, and since $R_{0}^{2}<2$, we obtain

$$
c_{0} \log \lambda<c R_{0}\left[1+\log \left(1+2^{3 / 2} \lambda\right)\right]
$$

this gives (6.15). 
7. Precise estimates for $\lambda$ large. We shall denote various positive constants independent of $\lambda$ by $C$.

LEMMA 7.1. If $\lambda$ is sufficiently large then

$$
d\left(B_{\lambda}\right) \leqslant C / \log \lambda
$$

in particular,

$$
R_{1}-R_{0} \leqslant C / \log \lambda
$$

Recalling (6.6) we conclude

Corollary 7.2. As $\lambda \rightarrow \infty$,

$$
R_{1} \rightarrow \sqrt{2}, \quad R_{0} \rightarrow \sqrt{2} .
$$

Proof of Lemma 7.1. Consider the family of straight lines $l_{r}, R_{0} \leqslant r<R_{1}$, each forming an angle $2 \pi / 3$ with the $r$-axis; $l_{r}$ cuts the $z$-axis at $z=r$. By (6.2) $l_{r}$ intersect $\bar{B}$ at the point $(r, 0)$. Denote by $\left(r^{*}, z^{*}\right)$ the first point of intersection of $l_{r}$ with $\bar{B}$, i.e., the segment $l_{r}^{*}$ from $(0, r)$ to $\left(r^{*}, z^{*}\right)$ lies outside $B$ and $\left(r^{*}, z^{*}\right) \in \partial B$. Then

$$
\psi(0, r)-\psi\left(r^{*}, z^{*}\right)=\int \frac{\partial}{\partial l_{r}} \psi d l
$$

and

$$
\psi(0, r)=0, \quad \psi\left(r^{*}, z^{*}\right)=\gamma+\frac{1}{2} W\left(r^{*}\right)^{2} \geqslant \frac{1}{2} W\left(r^{*}\right)^{2} \geqslant C W,
$$

where Lemma 6.7 was used $\left(r^{*} \geqslant R_{0} \geqslant C\right)$. Integrating (7.4) with respect to $r$, $R_{0}<r<R_{1}$, we get

$$
\begin{aligned}
C W\left(R_{1}-R_{0}\right) & \leqslant \int_{R_{0}}^{R_{1}} \int \frac{\partial \psi}{\partial l_{r}} d l d r \leqslant C\left(R_{1}-R_{0}\right)^{1 / 2}\left(\iint|\nabla \psi|^{2} d r d z\right)^{1 / 2} \\
& \leqslant C\left(R_{1}-R_{0}\right)^{1 / 2}\left(\iint \frac{1}{r}|\nabla \psi|^{2} d r d z\right)^{1 / 2}
\end{aligned}
$$

since $R_{1} \leqslant C$. Since the last integral is $\leqslant C E^{1 / 2}$, we get

$$
W\left(R_{1}-R_{0}\right)^{1 / 2} \leqslant C E^{1 / 2}
$$

Using finally (6.12) and (6.3), assertion (7.2) follows. Next we have to show that

$$
Z \leqslant C / \log \lambda \quad \text { where } Z=\sup \left\{z ;(r, z) \in B_{\lambda}\right\} .
$$

This follows by the same method of proof of (7.2), writing

$$
\psi(0, z)-\psi(\bar{r}, z)=\int_{0}^{\bar{r}} \frac{\partial}{\partial r} \psi(r, z) d r
$$

where the segment $\{(r, z) ; 0<r<\bar{r}\}$ lies outside $B$ and $(\bar{r}, z) \in \partial B, 0<z<Z$. Integrating with respect to $z$ we can now proceed as before to derive (7.5).

Lemma 7.3. Denote by $D$ the disc $(r-\sqrt{2})^{2}+z^{2}<1$. Then for all $\lambda$ sufficiently large

$$
\inf _{v} \int_{D \backslash B} \frac{|\nabla v|^{2}}{r} d r d z \leqslant \frac{1}{\pi} \frac{E+O(1)}{(\gamma+W)^{2}}
$$


where $v$ varies over the class of all functions in $H^{1}(D \backslash B)$ satisfying

$$
v=1 \text { on } \partial B, \quad v=0 \text { on } \partial D \text {. }
$$

Proof. Using $R_{1}^{2} \geqslant 2$ and Lemmas 6.2 and 6.3, we have

$$
2 W<W R_{1}^{2}<C \log \lambda,
$$

so that

$$
W<C \log \lambda \text {. }
$$

By Lemma 7.1, $B$ is contained in a rectangle $R_{0}<r<R_{1},|z|<Z$, where $R_{1}-R_{0}+Z \leqslant C_{0} /(\log \lambda)$. It follows that on $\partial B$,

$$
\psi=\gamma+W r^{2} / 2=\gamma+W+g(r)
$$

where $|g(r)| \leqslant C,\left|g^{\prime}(r)\right| \leqslant C \log \lambda$; we have used here (7.8). Set

$$
\bar{R}_{0}=R_{0}-C_{0} / \log \lambda, \quad \bar{R}_{1}=R_{1}+C_{0} / \log \lambda
$$

and extend $g(r)$ into $\bar{R}_{0} \leqslant r \leqslant R_{0}$ and into $R_{1}<r<\bar{R}_{1}$ in such a way that

$$
|g(r)| \leqslant C, \quad g\left(\bar{R}_{0}\right)=g\left(\bar{R}_{1}\right)=0, \quad\left|g^{\prime}(r)\right|<C \log \lambda .
$$

Let $h(z)$ be a function satisfying

$$
\begin{aligned}
h(z)=1 & \text { if }|z|<C_{0} / \log \lambda, \\
h(z)=0 & \text { if }|z|>2 C_{0} / \log \lambda, \\
|h(z)| \leqslant 1, & \left|h^{\prime}(z)\right|<C \log \lambda .
\end{aligned}
$$

Consider the function $g(r, z)=g(r) h(z)$ in the set $D_{0} \backslash B$ where $D_{0}$ is the rectangle $\bar{R}_{0}<r<\bar{R}_{1},|z|<2 C_{0} / \log \lambda$. We easily verify that

$$
\int_{D_{0} \backslash B} \frac{1}{r}|\nabla g|^{2} d r d z<C ;
$$

further, $g=0$ on $\partial D_{0}$ and

$$
\psi=\gamma+W+g \text { on } \partial B .
$$

Next, in view of Lemma 7.1,

$$
\begin{aligned}
\psi(r, z) & \sim r \sqrt{2} /\left|x-x^{0}\right| \quad\left(x^{0}=\left(r^{0}, 0, z^{0}\right), r^{0}=\sqrt{2}, z^{0}=0\right), \\
\nabla \psi & \sim \nabla\left(r \sqrt{2} /\left|x-x^{0}\right|\right)
\end{aligned}
$$

as $\lambda \rightarrow \infty$. Consequently we can extend the function $g(r, z)$ from $D_{0} \backslash B$ into $D \backslash B$ in such a way that the extended function $g$ satisfies: $g=\psi$ on $\partial D$,

$$
\int_{D \backslash D_{0}} \frac{1}{r}|\nabla g|^{2} d r d z<C .
$$

Now,

$$
\int_{H} \frac{1}{r}|\nabla \psi|^{2} d r d z=\frac{E}{\pi}
$$

and, consequently,

$$
\int_{D \backslash B} \frac{1}{r}|\nabla \psi|^{2} d r d z<\frac{E}{\pi} .
$$


By the Dirichlet principle we then deduce that

$$
\inf _{w} \int_{D \backslash B} \frac{1}{r}|\nabla w|^{2} d r d z \leqslant \frac{E}{\pi}
$$

for all $w \in H^{1}(D \backslash B)$ satisfying $w=\psi$ on $\partial B$ and on $\partial D$. Writing $w=g+\bar{w}$ and using (7.11), (7.12) and (7.14), we find that

$$
\inf _{\bar{w}} \int_{D \backslash B} \frac{1}{r}|\nabla \bar{w}|^{2} d r d z \leqslant \frac{E+O(1)}{\pi}
$$

for any $\bar{w} \in H^{1}(D \backslash B)$ satisfying $\bar{w}=\gamma+W$ on $\partial B, \bar{w}=0$ on $\partial D$. Setting finally $v=\bar{w} /(\gamma+W)$, the assertion of the lemma follows.

Denote the right-hand side of (7.6) by $\Gamma$. Inequality (7.6) means that

$$
\operatorname{Cap}_{D} B \leqslant \Gamma
$$

where the left-hand side stands for the capacity of the set $B$ with respect to $D$; the capacity is taken with respect to the operator $L$ (rather than the Laplacian).

We shall use the following result recently proved by Caffarelli and Friedman [10]: If $D_{0}$ is a fixed disc with center in $B$ and if $d(B) \leqslant C / \log \lambda$, then for all $\lambda$ sufficiently large,

$$
\frac{1}{[\sqrt{2}+O(1 / \log \lambda)]} \frac{2 \pi}{\log [2 \pi / d(B)]} \leqslant \operatorname{Cap}_{D_{0}} B .
$$

From Theorem 6.5 we deduce that

$$
\Gamma=1 / \pi(4 E+O(1))
$$

so that, by Lemma 6.1,

$$
\Gamma \leqslant 2 \sqrt{2} \pi /[\log \lambda+O(1)] .
$$

Using this in (7.17) (recall (7.16)) we obtain

$$
\log (1 / d(B)) \geqslant \log C \lambda^{1 / 2}
$$

Thus:

LEMMA 7.4. There holds

$$
d(B) \leqslant C / \lambda^{1 / 2}
$$

for all $\lambda$ sufficiently large.

Since $2 \pi|B|=1 / \lambda$, we then also have:

COROllary 7.5. There holds

$$
d(B) \geqslant c / \lambda^{1 / 2} \quad(c>0)
$$

for all $\lambda$ sufficiently large.

If we use (7.18) in (7.17) (recalling (7.16)), we obtain

$$
2 \log (2 \pi / d(B)) \geqslant 8 \sqrt{2} \pi^{2} E+O(1) .
$$

Comparing with (7.21), we find that

$$
E \leqslant\left(1 / 8 \sqrt{2} \pi^{2}\right) \log \lambda+C,
$$

which complements (6.3). 
We summarize:

THEOREM 7.6. For all $\lambda$ sufficiently large,

$$
\begin{gathered}
c \leqslant d\left(B_{\lambda}\right) / \lambda^{1 / 2}<C, \\
E=\left(1 / 8 \sqrt{2} \pi^{2}\right) \log \lambda+O(1)
\end{gathered}
$$

where $c, C$ are positive constants; further,

$$
W=E / 2+O(1), \quad \gamma=3 E / 2+O(1) .
$$

8. Asymptotic limit of $\zeta$ as $\lambda \rightarrow \infty$. For a solution $\zeta$ of (2.6), write

$$
\zeta_{\varepsilon}(r, z)=\varepsilon^{2} \zeta(\sqrt{2}+\varepsilon r, \varepsilon z) \quad \text { where } \varepsilon=1 / \sqrt{\lambda} .
$$

Similarly we write

$$
\tilde{\zeta_{\varepsilon}}(r, z)=\varepsilon^{2 \tilde{\zeta}}(\sqrt{2}+\varepsilon r, \varepsilon z)
$$

for any function $\tilde{\zeta}$ satisfying (2.1)-(2.4). Recall that

$$
E(\zeta) \geqslant E(\tilde{\zeta})
$$

We shall now express $E(\zeta)$ in terms of $\tilde{\zeta}_{e}$. We begin with

$$
G\left(r, z, r^{\prime}, z^{\prime}\right)=\frac{(\sqrt{2}+\varepsilon \bar{r})\left(\sqrt{2}+\varepsilon \bar{r}^{\prime}\right)}{4 \pi} \int_{-\pi}^{\pi} \frac{\cos \theta}{J^{1 / 2}} d \theta
$$

where

$$
r=\sqrt{2}+\varepsilon \bar{r}, \quad z=\varepsilon \bar{z}, \quad r^{\prime}=\sqrt{2} \varepsilon \bar{r}^{\prime}, \quad z^{\prime}=\varepsilon \bar{z}^{\prime}
$$

and

$$
\begin{aligned}
J & =\varepsilon^{2}\left(\bar{z}-\bar{z}^{\prime}\right)^{2}+(\sqrt{2}+\varepsilon \bar{r})^{2}+\left(\sqrt{2}+\varepsilon \bar{r}^{\prime}\right)^{2}-2(\sqrt{2}+\varepsilon \bar{r})\left(\sqrt{2}+\varepsilon \bar{r}^{\prime}\right) \cos \theta \\
& \equiv a-b \cos \theta, \\
a & =4+2 \sqrt{2} \varepsilon\left(\bar{r}+\bar{r}^{\prime}\right)+\varepsilon^{2}\left(\left(\bar{z}-\bar{z}^{\prime}\right)^{2}+\bar{r}^{2}+\bar{r}^{2}\right), \\
b & =4+2 \sqrt{2} \varepsilon\left(\bar{r}+\bar{r}^{\prime}\right)+2 \varepsilon^{2} \bar{r} \bar{r}^{\prime} .
\end{aligned}
$$

Now

$$
\begin{aligned}
\int_{-\pi}^{\pi} \frac{\cos \theta}{a-b \cos \theta} d \theta & =-\frac{2 \pi}{b}+\frac{a}{b} \int_{-\pi}^{\pi} \frac{d \theta}{a-b \cos \theta} \\
& =-2 \pi / b+2 a \pi\left(a^{2}-b^{2}\right)^{-1 / 2} / b
\end{aligned}
$$

and

$$
\begin{aligned}
\left(a^{2}-b^{2}\right)^{-1 / 2} & =[(1+O(\varepsilon)) /(2 \sqrt{2} \varepsilon)]\left[\left(\bar{z}-\bar{z}^{\prime}\right)^{2}+\left(\bar{r}-\bar{r}^{\prime}\right)^{2}\right]^{-1 / 2}, \\
a & =4+O(\varepsilon), \quad b=4+O(\varepsilon) .
\end{aligned}
$$

It follows that

$$
\int_{-\pi}^{\pi} \frac{\cos \theta}{a-b \cos \theta} d \theta=\frac{\pi(1+O(\varepsilon))}{\sqrt{2} \varepsilon\left[\left(\bar{z}-\bar{z}^{\prime}\right)^{2}+\left(\bar{r}-\bar{r}^{\prime}\right)^{2}\right]^{1 / 2}}
$$

Setting

$$
G^{*}\left(r, z, r^{\prime}, z^{\prime}\right)=2 \sqrt{2}\left[\left(r-r^{\prime}\right)^{2}+\left(z-z^{\prime}\right)^{2}\right]^{-1 / 2}
$$


we conclude that

$$
G\left(r, z, r^{\prime}, z^{\prime}\right)=\varepsilon^{-1} G^{*}\left(\bar{r}, \bar{z}, \bar{r}^{\prime}, \bar{z}^{\prime}\right)(1+O(\varepsilon))
$$

uniformly when $(\bar{r}, \bar{z})$ and $\left(\bar{r}^{\prime}, \bar{z}^{\prime}\right)$ vary in any disc $D_{R}: \bar{r}^{2}+\bar{z}^{2}<R^{2}$. In the sequel we choose $R$ so that

$$
\text { supp } \zeta \subset(R \varepsilon) \text {-neighborhood of }(\sqrt{2}, 0) ;
$$

notice that $R$ can be taken to be independent of $\lambda$ (by Theorem 7.6).

From (8.4) we have

$$
\begin{aligned}
r d r d z & =\sqrt{2} \varepsilon^{2} d \bar{r} d \bar{z}(1+O(\varepsilon)), \\
r^{\prime} d r^{\prime} d z^{\prime} & =\sqrt{2} \varepsilon^{2} d \bar{r}^{\prime} d \bar{z}^{\prime}(1+O(\varepsilon)) .
\end{aligned}
$$

Using this and (8.6), we get the following expression for $E(\zeta)$ :

$$
\varepsilon E(\zeta)=2 \pi \int_{D_{R}} \int_{D_{R}} G^{*}\left(r, z, r^{\prime}, z^{\prime}\right) \zeta_{\varepsilon}(r, z) \zeta_{\varepsilon}\left(r^{\prime}, z^{\prime}\right) d r^{\prime} d z^{\prime} d r d z+O(\varepsilon)
$$

Further, by (2.2)-(2.4),

$$
\begin{gathered}
\int_{D_{R}} \zeta_{\varepsilon}(r, z) d r d z \leqslant 1+O(\varepsilon), \\
0 \leqslant \zeta_{\varepsilon}(r, z) \leqslant 1, \quad \zeta_{\varepsilon}(r, z)=\zeta_{\varepsilon}(r,-z) .
\end{gathered}
$$

We introduce the functional

$$
E^{*}(\eta)=2 \pi \int_{D_{R}} \int_{D_{R}} G^{*}\left(r, z, r^{\prime}, z^{\prime}\right) \eta(r, z) \eta\left(r^{\prime}, z^{\prime}\right) d r^{\prime} d z^{\prime} d r d z
$$

and the class $\mathscr{B}$ of functions $\eta(r, z)$ satisfying

$$
\begin{gathered}
0 \leqslant \eta(r, z) \leqslant 1, \\
\operatorname{supp} \eta \subset D_{R}, \\
\eta(r, z)=\eta(r,-z), \\
2 \pi \int_{D_{R}} \eta(r, z) d r d z \leqslant 1 .
\end{gathered}
$$

Let $\eta \in \mathscr{B}$. By adding an $O(\varepsilon)$ function to $\eta$, we can write it as $\tilde{\zeta}_{\varepsilon}+O(\varepsilon)$ where $\tilde{\zeta}_{\varepsilon}$ has the form (8.2) (with supp $\tilde{\zeta}$ in $R \varepsilon$-neighborhood of $(\sqrt{2}, 0)$ ), and $\tilde{\zeta}$ is in $Q_{\lambda}$. Using (8.3) we deduce from (8.8) and the corresponding result for $\tilde{\zeta}$,

$$
E^{*}\left(\zeta_{\varepsilon}\right) \geqslant E^{*}(\eta)+O(\varepsilon) .
$$

Now take any limit $\eta^{*}$ of $\zeta_{\varepsilon}\left(\varepsilon=\varepsilon^{\prime} \rightarrow 0\right)$ in the weak star topology of $L^{\infty}$. Then we find, taking $\varepsilon=\varepsilon^{\prime} \rightarrow 0$ in (8.16), that $E^{*}\left(\eta^{*}\right)>E^{*}(\eta)$. From (8.9) and (8.10) it follows that $\eta^{*} \in \mathscr{B}$. Thus $\eta^{*}$ is the solution of the variational problem

$$
\eta^{*} \in \mathscr{B}, \quad E^{*}\left(\eta^{*}\right)=\max _{\eta \in \mathscr{B}} E^{*}(\eta)
$$

Recalling (8.5), it is clear that the only solution of (8.17) is

$$
\eta^{*}(\bar{r}, \bar{z})=I_{D_{0}}
$$


where $D_{0}$ is the disc $D_{0}: \bar{r}^{2}+\bar{z}^{2}<1 / 2 \pi^{2}$. We have thus proved

TheOREM 8.1. As $\lambda \rightarrow \infty$ the sets

$$
B_{\lambda}^{*} \equiv\left\{\left(\sqrt{2}+\lambda^{1 / 2} r, \lambda^{1 / 2} z\right) ;(r, z) \in B_{\lambda}\right\}
$$

converge to the disc $D_{0}$ with center at the origin and radius $1 /(\pi \sqrt{2})$; the convergence is in the sense that

$$
I_{B_{\lambda}^{*}} \rightarrow I_{D_{0}} \text { in the } L^{\infty} \text { weak star topology. }
$$

The convergence in (8.19) does not yet show that $\partial B_{\lambda}^{*}$ is approximately the disc $D_{0}$.

THEOREM 8.2. If we use polar coordinates $(\rho, \phi)$ about the center of $D_{0}$, then $\partial B_{\lambda}^{*}$ can be represented in the form $\rho=R_{\lambda}(\phi)(0<\phi<2 \pi)$ and, as $\lambda \rightarrow \infty$,

$$
\left(d^{i} / d \phi^{i}\right)\left(R_{\lambda}(\phi)-1 /(\pi \sqrt{2})\right) \rightarrow 0 \text { uniformly in } \phi \quad(i=0,1) .
$$

Proof. Introduce

$$
u_{\varepsilon}(r, z)=u(\sqrt{2}+\varepsilon r, \varepsilon z)
$$

Then

$$
\begin{gathered}
\Delta u_{\varepsilon}-[\varepsilon /(\sqrt{2}+\varepsilon r)]\left(u_{\varepsilon}\right)_{r}=-(\sqrt{2}+\varepsilon r)^{2} \zeta_{\varepsilon}(r, z), \\
u_{\varepsilon}=0 \text { on } \partial B_{\lambda}^{*}, \quad u_{\varepsilon}>0 \text { in } B_{\lambda}^{*}, \quad u_{\varepsilon}<0 \quad \text { in } R^{2} \backslash B_{\lambda}^{*} .
\end{gathered}
$$

We claim that if $r^{2}+z^{2}<\lambda / C_{0}$ (for a suitable $C_{0}>0$ ) then

$$
\left|u_{e}(r, z)\right| \leqslant C\left(r^{2}+z^{2}\right)^{1 / 2}+\lambda^{-1} C \log \lambda\left(r^{2}+z^{2}\right)^{1 / 2}+C .
$$

To prove it we write

$$
u_{\varepsilon}(r, z)=u_{\varepsilon}(r, z)-u_{\varepsilon}(\bar{r}, \bar{z}) \quad \text { for some }(\bar{r}, \bar{z}) \in \partial B_{\lambda}^{*},
$$

so that

$$
\begin{aligned}
u_{e}(r, z)= & {[\psi(\sqrt{2}+\varepsilon r, \varepsilon z)-\psi(\sqrt{2}+\varepsilon \bar{r}, \varepsilon \bar{z})] } \\
& +\left[\frac{1}{2} W(\sqrt{2}+\varepsilon r)^{2}-\frac{1}{2} W(\sqrt{2}+\varepsilon \bar{r})^{2}\right] \\
\equiv & I_{1}+I_{2} .
\end{aligned}
$$

From Lemma 2.3 or Lemma 3.3 in [15] (with obvious changes) we get

$$
|\nabla \psi|<C \lambda^{1 / 2}=C / \varepsilon
$$

where the argument of $\psi$ varies in the interval connecting $(\sqrt{2}+\varepsilon \overline{\mathbf{r}}, \varepsilon \bar{z})$ to $(\sqrt{2}+\varepsilon r, \varepsilon z)$. Hence

$$
\left|I_{1}\right| \leqslant C\left(r^{2}+z^{2}\right)^{1 / 2}+C
$$

Next,

$$
\left|I_{2}\right|<C \log \lambda \cdot \varepsilon r+C=\lambda^{-1 / 2} C \log \lambda r+C ;
$$

thus (8.23) follows. 
Let $U(R)\left(R=\left(r^{2}+z^{2}\right)^{1 / 2}\right)$ be the radial solution of

$$
\begin{gathered}
\Delta U=-2 I_{D_{0}}, \quad U=0 \quad \text { on } \partial D_{0}, \\
U=-A \log \left(R / R_{0}\right) \quad \text { if } R>R_{0}, \quad R_{0}=1 /(\pi \sqrt{2}) ;
\end{gathered}
$$

$A$ is uniquely determined by requiring that $U$ be $C^{1}$ at $R=R_{0}$; notice that $A>0$.

In view of (8.23) and (8.21), the standard elliptic theory shows that every sequence $\varepsilon_{j}^{\prime} \downarrow 0$ has a subsequence $\varepsilon_{j} \downarrow 0$ such that $u_{\xi_{j}} \rightarrow W$ uniformly in compact sets, together with the first derivatives, and, by Theorem 8.1,

$$
\Delta W=-2 I_{D_{0}}
$$

Also, by (8.23),

$$
|W(r, z)| \leqslant C\left(r^{2}+z^{2}\right)^{1 / 2}+C .
$$

From Theorem 8.1 we also deduce that

$$
\int_{B_{\lambda}^{*}} I_{A} u_{\xi_{j}} \rightarrow \int_{D_{0}} I_{A} W \quad\left(\varepsilon_{j}=\left(\lambda_{j}\right)^{-1 / 2}\right)
$$

for any characteristic function $I_{A}$. Since $u_{e_{j}}>0$ in $B_{\lambda j}^{*}$, we deduce that

$$
W \geqslant 0 \text { in } D_{0} \text {. }
$$

Similarly

$$
W \leqslant 0 \text { in } R^{2} \backslash D_{0}
$$

Near infinity $W$ is harmonic and thus has an expansion (we use here the estimate (8.26))

$$
W=a R \cos \left(\theta-\theta_{0}\right)+b \log R+\ldots
$$

In view of (8.28) it follows that $a=0, b \leqslant 0$. Thus

$$
W=b \log R+\ldots \quad \text { near } R=\infty .
$$

The properties (8.25) and (8.27)-(8.29) determine $W$ uniquely. Indeed, if $\bar{W}$ is another function satisfying all these properties then the function $Z=W-\bar{W}$ is harmonic and its first derivatives are bounded harmonic functions in $R^{2}$. Hence, by Liouville's theorem, $Z=$ const., i.e., $\bar{W}=W+c$. But the properties (8.27) and (8.28) for $\bar{W}$ then give $c=0$, so that $\bar{W} \equiv W$.

The function $W$ is clearly the same as the function $U$ defined above in (8.24). Thus we have proved that

$$
u_{e}(r, z) \rightarrow U(r, z), \quad \nabla u_{e}(r, z) \rightarrow \nabla U(r, z)
$$

uniformly in compact sets, where $U(r, z) \equiv U(R)$. Since $\partial U(r, z) / \partial R \neq 0$ on $R=R_{0}$, it follows that

$$
c \leqslant\left|\nabla u_{\varepsilon}(r, z)\right| \leqslant C \text { on } \partial B_{\lambda}^{*} .
$$

Further, if we represent $\partial D_{0}$ locally in the form, say, $r=k(z), k^{\prime}(z) \neq 0$, then we can represent $\partial B_{\lambda}^{*}$ locally in the form $r=k_{\lambda}(z)$ where

$$
k_{\lambda}(z)=-\frac{\partial u_{\varepsilon} / \partial z}{\partial u_{\varepsilon} / \partial r} \rightarrow-\frac{\partial U / \partial z}{\partial U / \partial r} \rightarrow k^{\prime}(z)
$$


$\left(\nabla u_{e}, \nabla U\right.$ are computed at the points $r=k_{\lambda}(z), r=k(z)$, respectively). The assertion of the theorem now readily follows. We have, incidentally, also proved:

COROllary 8.3. As $\lambda \rightarrow \infty, u(\sqrt{2}+\varepsilon r, \varepsilon z) \rightarrow U(r, z)$ with the first derivatives uniformly in compact subsets of $R^{2}$, where $U(r, z)=U(R)$ is the radial function given in (8.24).

REMARK. The asymptotic estimates of $\S \S 6-8$ can be extended also to the solution $\zeta_{\beta}=\zeta_{\beta, \lambda}$ of Theorem 2.2 when $\beta$ is fixed and $\lambda \rightarrow \infty$.

\section{REFERENCES}

1. J. F. G. Auchmuty, Existence of axisymmetric equilibrium figures, Arch. Rational Mech. Anal. 65 (1977), 249-261.

2. J. F. G. Auchmuty and R. Beals, Variational solutions of some nonlinear free boundary problems, Arch. Rational Mech. Anal. 43 (1971), 255-271.

3. G. K. Batchelor, An introduction to fluid dynamics, Cambridge Univ. Press, New York, 1974.

4. T. B. Benjamin, The alliance of practical and analytic insights into the nonlinear problems of fluid mechanics, Applications of Methods of Functional Analysis to Problems of Mechanics, Lecture Notes in Math., vol. 503, Springer-Verlag, Berlin, 1976, pp. 8-29.

5. H. Berestycki, Quelques questions liées à la théorie des tourbillons stationnaires dans un fluid ideal (to appear).

6. M. S. Berger and L. E. Fraenkel, On nonlinear desingularization, Bull. Amer. Math. Soc. (N.S.) 2 (1980), 165-167.

7. , Nonlinear desingularization in certain free boundary problems, Comm. Math. Phys. (to appear).

8. P. E. Byrd and M. D. Friedman, Handbook of elliptic integrals for engineers and scientists, Springer-Verlag, Berlin, 1971.

9. L. A. Caffarelli and A. Friedman, The shape of axisymmetric rotating fluid, J. Funct. Anal. 35 (1980), 109-142.

10. __ Asymptotic estimates for the plasma problem, Duke Math. J. 47 (1980), 705-742.

11. L. E. Fraenkel, On steady vortex rings of small cross-section in an ideal fluid, Proc. Roy. Soc. London Ser. A 316 (1970), 29-62.

12. _ Examples of steady vortex rings of small crass-section in an ideal fluid, J. Fluid Mech. 51 (1972), 119-135.

13. L. E. Fraenkel and M. S. Berger, A global theory of steady vortex rings in an ideal fluid, Acta Math. 132 (1974), 14-51.

14. A. Friedman and B. Turkington, Asymptotic estimate for an axisymmetric rotating fluid, J. Funct. Anal. 37 (1980), 136-163.

15. _ The oblateness of an axisymmetric rotating fluid, Indiana Univ. Math. J. 29 (1980), 777-792.

16. Existence and dimensions of a rotating white dwarf, J. Differential Equations (to appear).

17. H. Helmholtz, Über Integrale der hydrodynamischen Gleichungen, welche den Wirbelwegungen entsprechen, J. Reine Angew. Math. 55 (1858), 25-55.

18. M. J. M. Hill, On a spherical vortex, Philos. Trans. Roy. Soc. London Ser. A 185 (1894), 213-245.

19. H. Lamb, Hydrodynamics, Cambridge, 1932.

20. W. $\mathrm{M}$. $\mathrm{Ni}$, On the existence of global vortex rings (to appear).

Department of MATHEMATICS, NorthWESTERn UnIVERSTTY, Evanston, ILINOIS 60201 